\title{
Édition de la correspondance entre les folkloristes Walter Anderson et Joan Amades
}

\author{
Carme Oriol Carazo \\ Universitat Rovira i Virgili \\ carme.oriol@urv.cat
}

\begin{abstract}
RÉSUMÉ
Les folkloristes Walter Anderson (I885-I962) et Joan Amades (I89o-I959) ont maintenu une correspondance régulière au cours des dernières années de leur vie, alors qu'ils avaient tous deux publié leurs principales oeuvres et qu'ils se consacraient à de nouveaux projets. Dans ce contexte, l'objectif de ce travail est de divulguer toute cette correspondance transcrite dans la langue d'origine. L'édition de cette correspondance est accompagnée de notes explicatives dont le but est de documenter le contenu des lettres dans le contexte des études de folklore de l'époque.
\end{abstract}

MOTS CLÉS

Walter Anderson; Joan Amades; épistolaire; folklore; conte populaire; catalan

RESUM

Els folkloristes Walter Anderson (I885-I962) i Joan Amades (I890-I959) van mantenir una fluida correspondència en els darrers anys de la seva vida, quan tots dos havien realitzat les seves obres més importants i encaraven nous projectes. En aquest context, l'objectiu del present treball és donar a conèixer tota aquesta correspondència, transcrita en la llengua original. L'edició de la correspondència s'acompanya de notes explicatives que tenen com a finalitat documentar el contingut de les cartes en el context dels estudis de folklore de l'època.

PARAULES CLAU

Walter Anderson; Joan Amades; epistolari; folklore; rondalla; català 


\begin{abstract}
The folklorists Walter Anderson (I885-I962) and Joan Amades (I890-I959) maintained a regular correspondence during the last years of their lives, by which time both had completed their most important works and were looking to embark upon new projects. In this context, the present work aims to bring to light this correspondence, transcribed in their original language. The letters are published with explanatory notes that attempt to situate their content within the context of folklore studies at that time.
\end{abstract}

KEYWORDS

Walter Anderson; Joan Amades; collected letters; folklore; folktale; Catalan 


\section{Introduction ${ }^{1}$}

Le folkloriste allemand Walter Anderson (Minsk, IO.Io.I885 - Kiel, 23.08.I962) ${ }^{2}$ et le folkloriste catalan Joan Amades (Barcelone, 23.07.I890 - Barcelone, I7.OI.I959) ${ }^{3}$ commencèrent leur relation épistolaire le I8 janvier I953. ${ }^{4}$ Cette année-là, Anderson, qui avait obtenu l'adresse d'Amades à travers le folkloriste français Paul Delarue, écrivit la première lettre d'une correspondance qui allait durer entre les deux folkloristes jusqu'à fin I958. Après la mort d'Amades, en janvier 1959, Anderson continua à écrire à Enriqueta Mallofrè, veuve d'Amades, et à Consol Mallofrè, belle-sœur et collaboratrice de ce dernier. Enfin, après la mort d'Anderson en I962, les sœurs Mallofrè adressèrent une lettre de condoléances à Helene Anderson, épouse du folkloriste allemand. Cette lettre est datée du I2 septembre i962 et elle constitue la dernière de celles aujourd'hui publiées et dont les protagonistes sont Walter Anderson et Joan Amades.

La correspondance entre Anderson et Amades est conservée à Barcelone, au Centre de Documentation de la Culture populaire du Ministère de la Culture du Gouvernement de la Generalitat de Catalogne, ${ }^{5}$ et elle comprend 36 documents répartis de la manière suivante: 26 lettres et 5 cartes postales envoyées par Walter Anderson à Joan Amades (rédigées en français), 4 lettres envoyées par Walter Anderson à Enriqueta Mallofrè et Consol Mallofrè (également en français), et I lettre envoyée par les sœurs Mallofrè à Helene Anderson (rédigée en anglais). ${ }^{6}$

Compte tenu des possibilités que ce fonds documentaire offre à la recherche, l'objectif de ce travail est de mieux faire connaître le contenu de cette correspondance inédite jusqu'à nos jours, à l'aide de sa transcription dans les langues dans lesquelles elle a été rédigée à l'origine : le français et l'anglais. Notre objectif est donc de divulguer les rapports professionnels qui ont existé entre Joan Amades et Walter Anderson dans le cadre des études de folklore de l'époque. Pour atteindre cet objectif, la transcription est accompagnée de notes en bas de page qui permettent de documenter et d'expliquer les contenus de cette correspondance.

I. Ce travail s'est inscrit dans le cadre du projet «La literatura popular catalana en la segona meitat del segle Xx: documentació, estudi i difusió » (FFI2OI2-31808), financé par le Ministère de l'Économie et de la Compétitivité, et de les activités du Grup de Recerca Identitats en la Literatura Catalana (GRILC) (2OI4 SGR 755).

2. Au sujet de la vie et de l'œuvre de Walter Anderson, consulter Ranke (1977), Seljamaa (2005) et Järv (2008).

3. Au sujet de la vie et de l'œuvre de Joan Amades, consulter Calvo (I990) et Oriol (2008).

4. Pour d'autres études qui ont tenu compte d'informations contenues dans la correspondance entre les deux folkloristes, consulter Calvo (I990) et Oriol (I998, I999 et 200I).

5. Je remercie tout particulièrement Verònica Guarch, Antoni Serés, et Marcus Yáñez pour leur aide pour la consultation de la correspondance de Joan Amades qui est conservée au centre de documentation.

6. Ce fonds contient également une série de documents relatifs à la correspondance entre Anderson et Amades mais qui n'ont pas été publiés dans ce travail, car ils ne constituent pas une correspondance proprement dite. Ces documents sont les suivants : l'avis de décès de Walter Anderson, quatre brouillons de lettres des années I959 et I960 écrits en catalan par les sœurs Consol Mallofrè et Enriqueta Mallofrè et adressés à Walter Anderson, et le brouillon manuscrit, en catalan, de la lettre de condoléances que les deux sœurs envoyèrent, tapée à la machine en anglais, à Helene Anderson le I2 septembre I962. 


\section{Critères de l'édition}

L'ensemble des documents est présenté dans l'ordre chronologique. Chacune des entrées est identifiée à l'aide de sigles suivis d'un numéro corrélatif faisant référence à l'émetteur et au destinataire de la correspondance (A : Anderson ; Am : Amades; CM: Consol Mallofrè; EM : Enriqueta Mallofrè; M: les sœurs Enriqueta et Consol Mallofrè; et HA: Helene Anderson). Les sigles sont complétés par la date et le lieu de rédaction, puis, entre crochets, par les caractéristiques du document: s'il s'agit d'une carte postale ou d'une lettre, et si elle est manuscrite ou tapée à la machine. Le deuxième bloc de données correspond au format du contenu du document qui est reproduit avec la plus grande fidélité possible par rapport à l'original (y compris, par exemple, les nombreux termes soulignés qu'Anderson utilisait pour insister sur ce qu'il considérait important). Le respect des sources utilisées fait que les différences entre les originaux et les transcriptions sont minimes; en effet, ces différences se limitent essentiellement aux aspects typographiques comme, par exemple, le recours aux caractères en italique pour indiquer le titre d'une publication, la régularisation de la ponctuation et l'usage des majuscules. Enfin, la dernière ligne comprend la formule de conclusion et la signature.

La correspondance entre Anderson et Amades est répartie en trois sections dont le contenu est le suivant: (I) Correspondance de Walter Anderson à Joan Amades (identifiée par les sigles A-Am) et comprenant 26 lettres et 5 cartes postales écrites en français; (2) Correspondance de Walter Anderson à Consol Mallofrè et à Enriqueta Mallofrè (identifiée respectivement par les sigles A-CM et A-EM) et comprenant 4 lettres écrites en français; et (3) Correspondance des sœurs Enriqueta et Consol Mallofrè à Helene Anderson (identifiée par les sigles M-HA) et comprenant une seule lettre écrite en anglais. 


\section{La correspondance Anderson-Amades}

\subsection{Correspondance de Walter Anderson à Joan Amades}

[A-Am 1] 18.01.1953. Kiel (Allemagne, zone britannique), Forstweg 61. [lettre manuscrite]

Monsieur,

Permettez à un inconnu, qui prépare une grande monographie sur le célèbre conte des Trois oranges, ${ }^{7}$ de vous demander quelques renseignements. C'est $\mathrm{M}^{8}$ Paul Delarue ${ }^{9}$ qui m’a donné votre adresse.

Dans votre recueil monumental Folklore de Catalunya, ${ }^{\text {Io }}$ tome I, p. 23I, vous citez un texte de mon conte que je ne connais pas: Alcover, Les millors rondalles de Mallorca ${ }^{\text {II }}$ Ciutat de Mallorca, I947, p. 333. Je crois que ce n'est que la reproduction littérale d'un autre texte que je possède depuis quelques mois: Alcover, Aplech de rondayes mallorquines, ${ }^{12}$ tome III, Ciutat de Mallorca, I898, p. 65-IOI, «L'amor de les tres taronjes » (commencement: « Axò era un rey y una reyna que no tenían cap infant, y desitjavan ferm tenirne. Prometeren que, si 'n tenían un, donarían a cada pobre qui 's presentás una barsella de farina, una satría d'oli y una olleta de mel... »). —Ai-je raison, ou non? ${ }^{13}$

Puis, une autre question. Possédez-vous encore d'autres textes manuscrits du conte des Trois oranges, outre ceux que vous avez publiés dans votre recueil sous les numéros 62 et 93 ? $^{\text {I4 }} \mathrm{Si}$ c'était ainsi, je vous serais extrêmement reconnaissant si vous pouviez me les envoyer pour deux semaines: je les copierais et je vous les renverrais recommandés. Cette prière est peut-être trop hardie, mais je tâche de me procurer toutes les versions existantes de mon conte, et j'en ai recueilli déjà beaucoup plus que deux cents, provenant de l'Europe, de l'Amérique, de l'Afrique et de l'Asie (où le conte est connu jusqu'en Chine et Corée).

Il va sans dire que je comprends le catalan et l'espagnol, mais je ne connais pas ces deux langues assez bien pour en faire usage dans mes lettres.

En vous priant d'excuser mon audace, je reste votre très-dévoué. Walter Anderson, professeur de folklore à l'université de Kiel.

7. Le manuscrit de la monographie «The Three Oranges » [ATU 408], qui n'a jamais été publié, est conservé dans les archives de l'Enzyklopädie des Märchens (Göttingen, Allemagne).

8. J'ai maintenu la lettre « $\mathrm{M}$ » pour indiquer « Monsieur » comme le fait Anderson dans ses lettres.

9. Paul Delarue (Saint Didier, 20.04.I889 - Saint Didier, 25.07.I956). Au sujet de sa vie et de son œuvre, consulter Tenèze (I957, I958, I98I).

Io. Amades (I950).

II. Alcover (I946). La publication de Les millors rondalles de Mallorca remonte à I946 et non à 1947 comme indiqué dans la lettre. L'erreur est due à la référence qu'Amades donne de ce livre dans la bibliographie de son œuvre Folklore de Catalunya. Rondallística (RondallesTradicions-Llegendes) (Amades I950: I555).

I2. Alcover (I898).

I3. Effectivement, Anderson fait une bonne supposition. Il s'agit du même conte, comme l'atteste Guiscafrè (I999: I64).

I4. Il s'agit des contes «Les tres taronges de la vida» (Amades I950, n 62) et «L'amor de les tres taronges » (Amades I950, nº 93). 
[A-Am 2] 05.02.1953. Kiel, Forstweg 61. [lettre manuscrite]

\section{Cher Collègue,}

Je vous remercie cordialement de votre aimable lettre et du précieux texte que vous avez eu la bonté de faire copier pour moi.

Quant aux autres variantes catalanes que vous énumérez, je les possède toutes, avec une seule exception: Valeri Serra i Boldú, Rondalles populars, ${ }^{15}$ Barcelona I930, vol I, p. 45. C'est un livre dont l'existence m'était restée absolument inconnue, et que je n'ai pas l'espérance de trouver dans aucune bibliothèque allemande. Peutêtre la variante qui s'y trouve n'est que la reproduction littérale d'un autre texte que je connais déjà (Alcover, Maspons i Labrós, etc.). Si ce n'est pas ainsi, je vous serais infiniment obligé si vous faisiez copier pour moi ce texte aussi.

M. Delarue a découvert dans votre grand recueil et copié pour moi encore une troisième variante des «Trois oranges »: p. 278-279, $\mathrm{n}^{\circ}$ II6 « La reina cogullada ». ${ }^{\mathrm{I} 6}$ Le commencement du récit y est altéré, ainsi que les trois oranges en sont complètement disparues, mais la seconde moitié du conte est bien conservée.

Ce que vous m'écrivez sur le livre populaire catalan Les tres pomes de l'amor ${ }^{17}$ m'inspire un extrême intérêt. C'est sans aucun doute une variante du conte que j'étudie!

Quant aux trois volumes du recueil d'Espinosa, ${ }^{18}$ je ne les connais pas seulement, mais je les ai aussi dans ma bibliothèque.

En vous remerciant encore une fois et en vous priant d'excuser mon audace et mon insistance, je reste votre très-dévoué serviteur. Walter Anderson.

\section{[A-Am 3] 15.02.1953. Kiel, Forstweg 61. [lettre manuscrite]}

Cher Collègue,

Grand merci pour votre nouvelle lettre et pour le résumé du conte catalan, qui me serait resté inaccessible sans votre secours bienveillant! Je possède maintenant toutes les dix versions catalanes de mon conte dont l'existence m'est connue.

Votre résumé contient presque tous les détails de la version de Serra i Boldú, ${ }^{19}$ p. 45, qui aient pour moi quelque importance. Malheureusement, quelques points ne sont pas encore bien clairs, et je vous serais infiniment obligé, si vous pouviez répondre en quelques paroles aux questions suivantes:

I) Est-ce que le géant tient les yeux ouverts en dormant (et fermés en veillant), ou non?

I5. Serra i Boldú (I930-33, vol. I).

I6. Amades (I950, $\mathrm{n}^{\circ}$ II6).

I7. Je n'ai pas pu trouver ce livre auquel se réfère Anderson. Malgré tout, il pourrait s'agir de L'amor de les tres pomes. Rondalla [s.d.], un petit opuscule de quatorze pages publié à la fin du I $9^{\text {e }}$ siècle dans la collection Biblioteca infantil. Cependant, le conte ne correspond pas au type ATU 408 "L'amor de les tres taronges ", objet de la monographie d'Anderson, mais au type ATU 3OI «Les tres princeses robades » (Oriol-Pujol 2003 et 2008). Les contes suivants avaient été publiés auparavant dans cette même collection: Lo camí del cel, Los tres diables, La flor de Penical, Lo carboner, Lo país dels tontos, La Ventafocs, Lo raïm, Lo sac de farina, Lo Sarradell, Lo fill del diable et Lo sabater de baix.

18. Espinosa (1946-47).

I9. Serra i Boldú (I930-33, vol. I). 
2) Est-ce que la vieille sorcière enfonce l'aiguille enchantée dans une oreille de la fille, ou dans un autre endroit de sa tête?

3) Est-ce que la fille est métamorphosée en colombe, ou en un autre oiseau?

4) Est-ce que la sorcière promet au prince de devenir belle de nouveau, ou non?

5) Est-ce que l'oiseau vient trois fois dans le jardin du prince pour parler au jardinier, ou non?

6) Si c'est ainsi: quelles sont les paroles de l'oiseau et du jardinier?

7) Est-ce que les arbres où l'oiseau repose se dessèchent, ou non?

8) Est-ce que l'oiseau est attrapé dans un lacet (peut-être en employant un lacet d'or, un d'argent et un de diamants), ou avec de la glu?

9) Est-ce que l'oiseau salit l'assiette de la sorcière, ou non?

Io) Est-ce que la sorcière feint une maladie et demande la mort de l'oiseau (pour le manger), ou non?

II) Est-ce que la fille prononce le jugement de la sorcière (par exemple: « qu'on fasse de sa peau un tapis et de ses os une échelle ») ou la sorcière le prononce-t-elle (sans le savoir) elle-même?

I2) La sorcière est-elle brûlée, ou attachée à la queue d'un cheval, ou déchirée par quatre chevaux, etc.?

Comme je conserve une copie de cette lettre-ci, il suffit de répondre à ces questions tout laconiquement, par exemple: «I) Non. 2) Dans une tempe. 3) Colombe. 4) Non. 5) Oui », etc.

Vous avez déjà tant fait pour moi que je me sens tout embarrassé en vous adressant encore les questions ci-dessus (sûrement pour la dernière fois). Mais ce sont précisément ces petits détails qui me permettent de retracer l'histoire du développement et des migrations de mon conte.

Je vous remercie aussi de vos renseignements sur le livre populaire catalan. C'est stupéfiant, mais vous avez raison: ce n'est point le conte des « Trois oranges ", mais un autre conte (très répandu dans tout le monde); seulement le détail des trois pommes d'amour (au lieu du pommier à fruits d'or) est emprunté à mon conte. ${ }^{20}$

En vous remerciant de votre bonté et en vous priant d'excuser encore une fois mon audace, je reste votre très-dévoué. Walter Anderson.

\section{[A-Am 4] 26.02.1953. Kiel, Forstweg 61. [lettre manuscrite]}

\section{Cher Collègue!}

Je vous remercie le plus vivement de la réponse à mon questionnaire, et surtout des trois nouveaux textes catalans que vous avez eu la bonté d'y ajouter. Maintenant la tradition catalane de mon conte est très bien représentée dans mes matériaux: j'en possède déjà treize versions catalanes!

Si vous réussissiez à trouver des versions espagnoles inédites des «Trois oranges », je vous en serais très obligé tout de même. Quant aux versions espagnoles imprimées, j'espère de les connaître presque toutes (pour le moins celles qui ont été registrées par Espinosa).

20. Ce commentaire confirmerait l'hypothèse selon laquelle le livre auquel se réfère Anderson dans la lettre précédente (datée du 05.02.1953) est en réalité L'amor de les tres pomes. Rondalla [s.d.] [ATU 3OI]. 
Les organisations nordiques des folkloristes dont vous parlez ne sont pas des sociétés spéciales: ce sont des archives où tous les matériaux folkloriques d'une nation (imprimés ou manuscrits) sont recueillis et conservés. Il y a de telles archives à Copenhague, à Oslo en plusieurs endroits de la Suède, à Helsinki, à Tartu, à Riga. En Allemagne on peut mentionner les archives des chansons populaires à Fribourg et des contes populaires à Marbourg. Même la grande «Fédération des Folkloristes » internationale n'est pas une vraie société concrète: c'est plutôt une ligue idéale de tous les folkloristes du monde qui se sont promis de s'entr'aider dans leurs études.

Les « FF Communications ", qui sont éditées au nom de cette fédération par l'Académie Finnoise des Sciences, sont une série internationale où jusqu'à présent on a publié seulement des ouvrages écrits en allemand, en anglais ou en français. En Finlande il y a deux langues officielles (qui n'ont aucune ressemblance entre elles) : le finnois et le suédois ; et cependant ni le finnois et le suédois ne sont admis dans cette série! Peut-être on pourrait y publier aussi quelque chose en italien ; pour l'espagnol c'est déjà beaucoup plus douteux; mais un ouvrage catalan trouverait trop peu de lecteurs: la connaissance de votre belle langue est malheureusement trop peu répandue dans le monde.

La connaissance des langues étrangères c'est la plus grande difficulté pour chacun qui veuille écrire une monographie sur un conte populaire. Il doit connaître au moins toutes les langues romanes, germaniques et slaves (non seulement les langues littéraires, mais aussi les différents dialectes) ou bien se faire traduire les textes écrits en ces langues, ce qui coûte beaucoup d'argent. A cet égard, moimême je suis assez heureux: je puis déchiffrer des textes écrits en plus de quarante langues (y compris le finnois, l'estonien, le hongrois, l'hébreu, l'arabe, le persan et le turc).

Quant à la brochure que je vous ai envoyée, elle est intitulée « Une expérience folklorique $»{ }^{21} \mathrm{Il}$ y a plus de trente ans, j'avais établi la thèse que chaque homme qui récite un conte populaire l'a entendu lui-même non pas une seule fois dans sa vie, mais presque toujours plusieurs fois et de la bouche de plusieurs personnages; autrement le conte s'altérerait et se déformerait de plus en plus, ainsi que bientôt il ne serait plus à reconnaître. Cette thèse fut combattue par beaucoup de folkloristes, qui ne voulaient pas y croire. Pour décider la question, je lus une légende (peu connue) à trois étudiants de notre université, en les priant de l'écrire le jour suivant ainsi comme ils s'en souviendraient alors, et de la lire chacun à un autre étudiant, qui un jour après ferait la même chose; et ainsi de suite douze fois. En résultat j'eus trois séries, de i2 textes chacune, et les derniers textes de ces séries ( $\mathrm{AI}_{2}, \mathrm{BI}_{2}, \mathrm{C}_{12}$ ) étaient tous affreusement déformés et divergeaient entre eux complètement; c'était précisément ce que j'avais prévu. Il y a une vingtaine d'années, j'avais fait quelques expériences analogues en Estonie (sans en publier les résultats); les résultats avaient été absolument identiques.

Je ne pourrai commencer à écrire ma monographie sur les « Trois oranges » avant la fin de cette année, parce que je suis encore à la recherche de quelques textes importants publiés dans des livres peu accessibles. C'est pourquoi je puis encore utiliser tous les matériaux que je trouverais encore ou qui me seraient envoyés.

2I. Anderson (I95I). 
Si vous m'envoyiez quelqu'un de vos nouveaux ouvrages, ce serait pour moi un grand plaisir d'en publier une critique dans quelque revue folklorique allemande ou suisse.

En vous remerciant encore une fois de votre obligeance, je reste votre très-dévoué. Walter Anderson.

\section{[A-Am 5] 23.03.1953. Kiel, Forstweg 61. [lettre manuscrite]}

Cher Collègue,

Je viens de recevoir votre lettre du 20 mars. C'est bien étonnant que vous n'ayez pas reçu celle que je vous avais adressée le 26 février. Comme j'en ai conservé le brouillon, je puis vous envoyer ci-joint une copie complète de cette lettre perdue. $^{22}$

Après avoir reçu l'ouvrage que vous avez l'obligeance de me promettre, ${ }^{23}$ j'en publierai une critique dans la Zeitschrift für Volkskunde.

Je suis toujours votre très-dévoué. Walter Anderson.

\section{[A-Am 6] 04.04.1953. Kiel, Forstweg 61. [lettre manuscrite]}

Cher Collègue,

Aussitôt après avoir expédié ma dernière carte postale, j'ai reçu votre lettre du 30 mars.

La Fédération des Folkloristes qui vous intéresse est une organisation bien étrange: une organisation sans président, sans commissions, sans filiales, sans budget, sans séances, sans procès-verbaux, sans comptes-rendus. Presque toutes ces choses avaient été projetées au temps que la fédération fut fondée (en I907), mais elles ne se sont pas réalisées, parce qu'on les a regardées comme superflues. La Fédération est quelque chose comme la "république des savants » imaginée par Erasme de Rotterdam: on en devient membre en déclarant (ou même en montrant) qu'on est prêt à aider les autres folkloristes dans leurs études —et maintenant vous en êtes membre au même titre que moi. Si quelque folkloriste a besoin de matériaux folkloriques catalans, je lui donnerai votre adresse —de même que je l'ai reçue de la part de M. Paul Delarue. Et si vous-même vous aurez besoin de quelques matériaux, de renseignements bibliographiques etc., moi et tous les autres folkloristes, nous serons heureux de pouvoir vous servir à cet égard. C'est tout; il n'y a pas de formalités, il n'y a pas de listes de membres. -Moi-même, je suis membre du comité de rédaction des FF Communications ${ }^{24}$ (le seul comité de la Fédération!), c'est-à-dire de la série éditée au nom de la Fédération des Folkloristes et financée par l'Académie Finnoise des Sciences; depuis I926 mon nom, à côté de cinq autres noms, figure sur les couvertures de tous les volumes de cette série (il y en a déjà 6o, formés par I44 numéros); et cependant ni moi ni mes cinq

22. La lettre (datée du 26.02.I953) se trouve à l'endroit correspondant, conformément au critère de publication retenu qui est chronologique.

23. Anderson se réfère à Les cent millors rondalles populars (Amades I948-49) ou à la deuxième édition de cette même œuvre (Amades I953), comme on peut le déduire d'une lettre ultérieure (datée du oi.IO.I953).

24. Au sujet de la collection Folklore Fellows Communications, consulter Pentikäinen (I982). 
collègues (parmi lesquels le prof. Martti Haavio ${ }^{25}$ est le chef) ne possédons aucun document qui atteste notre qualité de rédacteurs (ou même de membres de la Fédération)!

Quant à vos observations sur la transmission des contes (et des chansons) populaires, je suis de votre avis: ce sont presque les mêmes observations que j'ai faites moi-même.

Je suis bien étonné de lire que vous avez recueilli tant de contes et de chansons populaires: I5.000 — c'est un nombre colossal!

Je reste toujours votre très-dévoué serviteur. Walter Anderson.

\section{[A-Am 7] 01.10.1953. Kiel, Forstweg 61. [lettre manuscrite]}

Cher Collègue,

Je me hâte de répondre à votre lettre du 28 septembre, dont je vous remercie vivement.

Le conte roussillonnais d'Horace Chauvet $^{26}$ (qui est plutôt un bref résumé) m'a déjà été communiqué par M. Delarue.

Vous parlez du congrès de Namur, et vous regrettez de n'avoir pu y aller; vous ne m'y auriez pas rencontré, car moi non plus je n'y ai pu prendre part.

Quant à la définition de ce qui est un conte populaire, je confesse de me soucier assez peu de définitions et classifications - en tout cas moins que vous qui êtes un compatriote de Raymond Lulle. Dans l'univers il y a tant de choses qui résistent à une définition rigoureuse, tant de formes de transition ou hybrides qui ne peuvent être classées. Moi j'appellerais un « conte » tout récit de ce qui s'est passé une fois, mais non pas de ce qui se passe beaucoup de fois à présent. Bien des peuples sauvages croient que pendant chaque éclipse le soleil ou la lune sont dévorés par un monstre et sortent puis de son ventre: ce n'est pas un conte, c'est une croyance. Le peuple russe dit que la terre repose sur trois baleines gigantesques, dont les convulsions causent les tremblements de terre: cela aussi, c'est une croyance, ce n'est pas un conte. Mais il y a aussi des peuples qui croient qu'au temps jadis il y a eu au ciel dix soleils qui brûlaient la terre sans pitié, jusqu'à ce qu'un héros en tua neuf par ses flèches: ceci, c'est un conte véritable —un conte étiologique.

Il y a des contes qui expliquent l'origine d'un proverbe ou d'une devinette, ou bien d'un surnom moqueur: tout cela, ce sont toujours des contes; mais par exemple les jeux de doigts ne le sont pas: ce sont plutôt de petits drames que de contes. Je ne classerais pas parmi les contes non plus les problèmes mathématiques (par exemple celui des Ioo oies: $x+x+1 / 2 x+1 / 4 x+1=I O O)$, ni les textes que vous appelez « embarrassallengues ».

25. Martti Haavio (Temmes, 22.or.I899 - Helsinki, O4.O2.I973). Pour de plus amples informations sur son activité de folkloriste, consulter Honko (I988).

26. Il s'agit vraisemblablement du conte «Les trois oranges de l'amour » [ATU 4O8] (Chauvet I947: 24I-242). 
Quant au Motif-Index de M. Thompson, ${ }^{27}$ il registre (au contraire de ses Types of the Folk-Tale) ${ }^{28}$ non seulement des contes complets, mais aussi d'innombrables motifs et éléments de contes, qui eux-mêmes ne sont point des contes, mais plutôt des croyances etc.: par exemple l'existence de fées, d'ogres, etc.

J'ai lu les deux volumes de votre beau petit recueil ${ }^{29}$ et j'ai pris de nombreuses notes là-dessus, mais je n'ai pas encore eu le temps d'écrire la critique que je vous ai promise, empêché que j'étais par d'autres travaux pressants. Pourtant soyez sûr que je remplirai ma promesse, et souvenez-vous parfois de votre très-dévoué. Walter Anderson.

\section{[A-Am 8] 11.02.1954. Kiel, Forstweg 61. [lettre manuscrite]}

\section{Cher Collègue!}

Je regrette de ne pas avoir encore publié le compte-rendu de votre anthologie que je vous avais promis; c'est que je me suis décidé à en écrire un sur votre grand recueil de 1735 pages $^{30}$ que j'ai fait venir de l'Espagne.

A présent je suis à étudier cet incomparable recueil, dont le compte-rendu occupera bien une dizaine de pages. Il va sans dire que j'y mentionnerai aussi votre anthologie (en deux volumes).

La lecture de votre grand livre me fournit beaucoup de surprises. En lisant le conte $\mathrm{N}^{\circ} 23 \mathrm{I}$ " L'home tornat ruc ", ${ }^{\text {I }}$ je fis vraiment " des yeux comme des oranges »: c'est un conte répandu en Allemagne depuis la Guerre des Trente Ans (parmi ses versions, il y en a deux du XVII ${ }^{\mathrm{e}}$ siècle), un conte qui est probablement emprunté au roman d'Apulée, et que je connaissais très bien, puisque je l'avais étudié il y a plus de quarante ans! Mais je n'avais pas la moindre idée que ce conte fût connu aussi chez d'autres peuples —et surtout chez les Catalans!

Je désirerais consacrer à ce conte plusieurs pages dans quelque revue folklorique, en y ajoutant la traduction complète de votre texte catalan; ${ }^{32}$ mais alors j'aurais besoin aussi de l'autre version catalane que vous citez: Bertran, Ron-

27. Thompson (I932-36). Une deuxième édition corrigée et complétée du Motif-index of folk literature a été publiée en 1955-58.

28. Aarne-Thompson (1928).

29. Il s'agit de Les cent millors rondalles populars (Amades I948-49) ou de la deuxième édition de cette même œuvre, publiée en I953. Anderson lui avait promis d'en faire un compte rendu dans la lettre qu'il lui avait écrite le 23 mars 1953.

30. Il s'agit de l'œuvre Folklore de Catalunya. Rondallística (Rondalles-Tradicions-Llegendes) (Amades I950). Anderson décida de faire le compte rendu de cette œuvre qui contenait l'ensemble des contes d'Amades au lieu des deux volumes de Les cent millors rondalles populars qui n'étaient que l'anthologie.

3I. Le conte est très connu mais il n'a pas d'entrée dans l'index typologique international (Uther 2004). Par contre, dans le cadre du projet de catalogage des contes catalans effectué par les chercheurs des Archives de Folklore de l'Université Rovira i Virgili <http://www. arxiudefolklore.cat/>, nous lui avons affecté son propre numéro : C-o65 (consulter le site web « RondCat. Cercador de la rondalla catalana » http://www.sre.urv.cat/rondcat/).

32. Ce travail d'Anderson est à l'origine de deux publications: Anderson (I954a) et Anderson (I958). Dans cette deuxième publication, Anderson (I958: I2I) fait référence à I9 versions modernes du conte, dont quatre versions catalanes sont citées. 
dallari,45 = Rondalles, I, 45 (je crois que c'est un seul texte, et non pas deux). ${ }^{33}$ Pourtant je crains que ces deux livres ne sont pas accessibles en Allemagne; je tâcherai de me les procurer, mais je ne sais pas si j'y réussirai.

Je me permets de vous envoyer mon compte-rendu du grand catalogue des contes populaires turcs (publié par Eberhard et Boratav). ${ }^{34}$

Veuillez croire à l'expression des sentiments les plus respectueux de votre trèsdévoué. Walter Anderson.

\section{[A-Am 9] (s.d.) [1954] ${ }^{35}$ [lettre manuscrite]}

Cher Collègue,

Grand merci pour votre aimable lettre! J'espère que vous ayez reçu aussi mon article sur l'homme transformé en âne, que je vous ai envoyé il y a quelques jours.

Vous avez raison: la composition d'un catalogue national de contes populaires est un travail extrêmement utile, mais aussi très grand et très difficile.

Il faut enregistrer, si c'est possible, tous les contes populaires, bien qu'ils aient été publiés dans des almanachs, des revues, des journaux etc., et même ceux qui sont restés non-publiés; aussi ceux qui furent imprimés dans des livres d'enfants, pourvu que ce ne soient pas des remaniements artificiels de textes déjà enregistrés dans votre catalogue.

Pourtant il ne faut pas tenir compte des légendes locales sur les fées, les géants, les sirènes etc., sur les pays et les vaisseaux fabuleux, ni des légendes sur les vies des saints ou sur des miracles religieux. Ce sont là des choses très intéressantes, mais qui exigent un catalogue spécial.

Quant aux anecdotes et facécies, il faut en enregistrer le plus grand nombre possible - au moins toutes celles qui sont nommées dans le catalogue d'Aarne et Thompson. Pourtant les anecdotes purement locales (sur le blason populaire, etc.) pourraient être omises, de même que les interprétations du chant des oiseaux et les problèmes mathématiques.

En lisant votre grand recueil, je n'ai point enregistré tous vos contes, mais seulement ceux qui figurent dans le catalogue d'Aarne et Thompson, et ceux qui y manquent, mais sont d'ailleurs très connus et importants. Tout ceci j'ai imprimé dans mon compte-rendu.

Si vous avez des doutes sur quelque conte populaire, envoyez-m'en un bref résumé ou bien le numéro correspondant de votre Rondallistica, ${ }^{36}$ et je vous en dirai mon opinion.

Mille saluts de votre très-dévoué. Walter Anderson.

33. Il s'agit effectivement d'un seul texte, «El ruc de les bruixes » [C-065], publié dans Les rondalles populars catalanes (Bertran I908: 45-48) et dans El rondallari català (Bertran I908-09, vol. I: 45-48).

34. Anderson (1953).

35. Lettre sans indication de la date ni du lieu. Dans la correspondance Anderson-Amades, la lettre est archivée dans le dossier correspondant à l'année I954.

36. Amades (I950). 
[A-Am 10] 23.02.1954. Kiel. [carte postale manuscrite]

\section{Cher Collègue,}

Je vous remercie le plus vivement du nouveau texte que vous avez eu la bonté de m'envoyer, et qui sans votre secours me serait resté inaccessible. ${ }^{37}$

Je suis toujours à étudier votre inépuisable recueil : plus j'y lis — plus je l'admire.

Votre très-dévoué. Walter Anderson.

\section{[A-Am 11] 01.09.1954. Kiel. [lettre manuscrite]}

Monsieur le Collègue,

Grand merci pour votre lettre du 27 août et pour les deux nouvelles versions de mon conte! - La version de Milá y Fontanals m'est connue.

Les « Archives suisses des traditions populaires » où j'ai publié mon compterendu $\mathrm{u}^{38}$ sont voués au folklore et à l'ethnographie de tous les pays. Les travaux qui s'y publient sont rédigés en allemand, en français ou en italien. Je ne sais pas si l'on y admettrait aussi un article écrit en espagnol; en tout cas, vous pouvez vous adresser directement au rédacteur: Dr. Robert Wildhaber, Peter Ochs-Strasse 87, Bâle 24, Suisse.

Quant au livre d'Aarne-Thompson, je ferai tout mon possible pour vous le procurer dans quelques semaines.

Vous serait-il possible de m'envoyer le texte complet de ce que Maspons i Labrós dit sur le prétendu homme-âne dont il a fait la connaissance (Lo Gay Saber, segona època, any III, page —? année de publication —?)?39 J'ai déjà achevé le manuscrit de mon petit travail sur ce conte, et j'espère qu'il sera publié bientôt.

Ayant trouvé votre nom dans la liste des membres du congrès de Naples, ${ }^{40}$ j'espère y faire votre connaissance personnelle. Au cas qu'il n'y aura pas d'obstacles imprévus, j'y viendrai moi aussi.

Je reste votre très-dévoué. Walter Anderson

[A-Am 12] 16.10.1954. Kiel, Forstweg 61. [lettre manuscrite]

\section{Cher Collègue,}

Grand merci pour les trois versions inédites des « Trois oranges »! Je ne les publierai pas, mais j'en ferai usage en analysant ce conte pour en reconstruire

37. Il s'agit probablement de la version de Bertran «El ruc de les bruixes » citée par Amades dans une note sur "L'home tornat ruc » (Amades I95I, n² 23I), version à laquelle s'intéresse Anderson dans sa lettre du II février 1954.

38. Anderson (I954b).

39. Il s'agit d'une version de type "L'home tornat ruc » (RondCat C-065) que Maspons a inclus dans l'article «Las bruixas » publié dans Lo Gay Saber (Maspons I880). Concrètement, la version à laquelle se réfère Anderson apparaît dans la dernière partie de l'article, publié dans le numéro 24 du magazine.

40. Il pourrait s'agir du Congresso Internazionale di Etnografia e Folklore del Mare de Naples de I954, même si je n'ai pas trouvé suffisamment d'indices pour le corroborer. 
la forme originale et l'évolution postérieure. Il va sans dire que je mentionnerai aussi les Archives Historiques Municipales de Barcelone. ${ }^{4 \mathrm{I}}$

Ci-joint une traduction approximative des parties les plus importantes de mon compte-rendu. ${ }^{42}$

Mille saluts de votre très-dévoué. Walter Anderson

C'est bien connu que les Catalans ont été très actifs en recueillant leur contes populaires. La série de leurs recueils commence avec Milá y Fontanals; quant aux autres, je mentionne seulement Maspons y Labrós, Bertrán y Bros, l'archiduc Louis Salvator et Alcover. Le recueil de ce dernier est les plus volumineux; dans sa dernière édition il consiste de I4 volumes. Pourtant les textes d'Alcover, dont plusieurs sont très longs, éveillent quelquefois le soupçon d'avoir subi un remaniement littéraire.

Maintenant j'ai devant mes yeux un recueil de contes et légendes, qui surpasse de beaucoup tout ce qu'il y a eu jusqu'à présent: I735 pages de I4X2O cm, deux colonnes (excepté l'introduction), chaque colonne à 53 lignes, très petits caractères (qui exigent malheureusement de bons yeux ou de bonnes lunettes), très mince papier, une belle reliure en cuir, de bord supérieur doré. Et le nombre des contes, anecdotes et légendes monte à 22I5 (pardon, ce n'est pas une erreur typographique!); mais en réalité ce nombre est encore plus grand : non seulement quelques numéros (par exemple 465, I753 et I945) figurent deux fois chacun, mais sous beaucoup de numéros on trouve réunis plusieurs contes différents. Chaque conte est raconté tout simplement, sans aucun remaniement littéraire, et presque toujours pourvu d'indications concernant le nom du conteur, le lieu et l'année où le conte fut recueilli, et quelquefois des notices bibliographiques. Quant aux conteurs les plus importants (il y en a plus de 250), le lecteur en trouve des données bibliographiques aux pages I545 à I553. En tête de beaucoup de contes il y a une belle vignette dessinée par Francesc Almuni.

Et cependant ce livre imposant ne contient qu'une partie des innombrables matériaux recueillis par l'auteur!

[Mention de l'anthologie en 2 volumes. - Table des matières]

Nous voyons qu'il s'agit ici d'une publication folklorique extrêmement importante —d'un trésor de contes populaires qui ne doit être ignoré par aucun spécialiste. Mais c'est précisément la richesse fantastique du recueil catalan qui en rend l'usage difficile. Si je voulais donner un bref résumé de chacun des 22I5 numéros (comme c'est l'usage depuis Reinhold Köhler, Bolte et Polívka), je devrais écrire toute une brochure de dimensions considérables; donc, je dois y renoncer. Mais si un spécialiste se met à y chercher des versions d'un certain conte populaire - comment les pêchera-t-il de l'océan du livre catalan? Des versions d'un même conte s'y trouvent souvent dispersés en différents lieux — par exemple de véritables

4I. Pendant ces années, Amades travaillait aux Archives historiques municipales de la ville de Barcelone (Calvo I990: I88).

42. Anderson (I954b). 
contes de fées (grâce à une localisation accidentelle) dans le chapitre des légendes de châteaux. Est-ce que le spécialiste doit imiter mon exemple en sacrifiant plusieurs semaines à l'étude du livre catalan? Ce n'est pas une chose que chacun puisse faire! Pour remédier, au moins en partie, à ce mal, j'indique dans la table suivante les numéros, sous lesquels le lecteur trouvera les contes-types registrés par Aarne et Thompson.

[A-Am 13] 22.10.1956. Kiel. [lettre manuscrite]

Cher Collègue,

Ci-joint je vous envoie les données que vous désirez.

J'attends votre manuscrit français.

Votre très-dévoué. Walter Anderson.

[A-Am 14] 10.04.1957. Kiel. [carte postale manuscrite]

Cher Collègue,

C'est avec le plus grand plaisir que je classifierai les contes de tous les recueils que vous aurez la bonté de m'envoyer; c'est que votre Rondallística ${ }^{43}$ est le seul recueil catalan que les bibliothèques de notre ville possèdent.

En général, je ferai tout mon possible pour vous secourir dans votre entreprise. ${ }^{44}$

Votre très-dévoué. Walter Anderson.

[A-Am 15] 02.05.1957. Kiel. [carte postale manuscrite]

Cher Collègue,

Grand merci pour les quatorze volumes d'Alcover! ${ }^{45}$ Pourtant, il me faudra quelques semaines pour lire et classifier tous ces longs contes.

J'ai reçu les volumes I-VII et IX-XV; il faut croire que le volume VIII est épuisé; mais je pourrai le faire venir de la «Lateinamerikanische Bibliothek » à Berlin (qui possède les volumes I-XIV).

Votre très-dévoué. Walter Anderson.

43. Amades (I950).

44. À cette époque, Amades aurait initié le projet de catalogage des contes catalans qui, selon Calvo (I990: I72, I89), lui aurait été commandé par l’UNESCO.

45. Anderson se réfère aux quatorze premiers volumes des contes publiés par Alcover (193672 ). Au sujet des démarches effectuées par Amades pour obtenir ces volumes qu'il a envoyés à Anderson, consulter Oriol (200I). 
[A-Am 16] 07.08.1957. Kiel. [lettre manuscrite]

\section{Cher Collègue,}

Je vous envoie ci-joint mon analyse des contes d'Alcover. ${ }^{46}$ Je ne possède pas le huitième volume de ce recueil, mais il me fut prêté par la Bibliothèque LatinoAméricaine de Berlin.

Les légendes religieuses, étiologiques, historiques, etc. des tomes IV, V, VI et $\mathrm{XV}$, que j'ai marqué comme exclues du catalogue AaTh, ne doivent pas entrer dans votre catalogue.

Grand merci pour votre carte postale et surtout pour votre livre qui n'est pas encore arrivé, mais qui arrivera sans doute dans quelques jours!

Votre très-dévoué. Walter Anderson.

[A-Am 17] 25.08.1957. Kiel. [lettre tapée à la machine]

\section{Cher Collègue,}

Je vous remercie de votre aimable lettre que j'ai reçue avant-hier.

Vous me priez de vous expliquer bien clairement, quels contes sont exclus du système d'Aarne-Thompson. Hélas! C'est presque impossible; il faudrait un nouveau Raymond Lulle pour vous fournir toutes les définitions, distinctions et classifications que vous désirez. On peut seulement dire en général que les légendes religieuses, bibliques, étiologiques, historiques et locales sont exclues de ce système-là; pour tous les contes de ces genres on compose des catalogues à part. Ou bien, si c'est plus clair: vous avez à enregistrer dans votre catalogue seulement des contes tels que ceux que vous avez publiés dans votre Rondallística ${ }^{47}$ aux pages I-797, IO28-IO46 (surtout les légendes joyeuses!) et I047-IO69. Si un conte éveille vos doutes, envoyez-m'en un bref résumé, et je vous répondrai.

Les numéros des types qui manquent dans FFC $74^{48}$ seront fixés par M. Thompson ${ }^{49}$ lui-même qui prépare maintenant une nouvelle édition de son registre. ${ }^{50}$

Pour faciliter votre travail, j'ai l'intention de faire une révision de votre Rondallística ${ }^{5 \mathrm{I}}$ pour compléter l'index que j'en ai publié dans les Archives suisses des traditions populaires..$^{2}$

J'attends avec grand intérêt les recueils de contes catalans que vous avez eu la bonté de m'annoncer. ${ }^{53}$

Votre très-dévoué. Walter Anderson.

46. La lettre est accompagnée de I8 pages numérotées et contenant la classification des quinze premiers volumes des contes d'Alcover (1936-72).

47. Amades (I950).

48. Aarne-Thompson (I928).

49. Stith Thompson (07.03.I885 - I3.0I.I976) a été professeur de l'Université d'Indiana, Bloomington (USA) de I92I jusqu'à son départ en retraite, en I956.

50. La nouvelle édition du catalogue a été publiée quatre ans plus tard sous le titre The types of the folktale (Aarne-Thompson I96I).

5I. Amades (I950).

52. Amades (1954b).

53. Au sujet des contacts maintenus par Amades avec les folkloristes catalans pour obtenir des volumes de contes qu'il enverra plus tard à Anderson, consulter Oriol (I999). 
[A-Am 18] 12.09.1957. Kiel, Forstweg 61. [lettre manuscrite]

\section{Cher Collègue,}

Je vous remercie le plus vivement de votre précieux paquet, et surtout du gran recueil de Serra i Boldú que je n'avais encore jamais vu. ${ }^{54}$ Des dix-huit volumes de ce recueil, ce ne sont que les volumes VII, X, XII, XIII qui me manquent maintenant; je serais très heureux si vous trouviez l'occasion de me les procurer (de même que le volume VIII d'Alcover).

Quant à vos propositions, je ferai avec le plus grand plaisir tout le possible pour vous aider dans votre entreprise, à laquelle je prends beaucoup d'intérêt. Envoyez-moi toutes les fiches et toutes les questions que vous voulez, et j'arrangerai et j'expliquerai tout ce que je pourrai. Pourtant je ne puis consentir à ce que mon nom apparaisse à côté du vôtre sur le frontispice de votre catalogue: ce serait possible seulement si nous pouvions entretenir un contact quotidien et conférer personnellement sur toutes les questions qui s'élèvent pendant un tel travail; quant à la classification des contes populaires, il y a des points à l'égard desquels les plus grands spécialistes ne sont pas d'accord l'un avec l'autre. Je devrais donc contrôler tout votre travail, ce qui est impossible. - Mais vous pouvez, sans doute, mentionner et préciser ma collaboration dans la préface de votre livre.

Quant aux catalogues nationaux de légendes étiologiques, historiques, etc. on les a publiés pour la plupart en appendice aux catalogues des contes populaires. Je puis vous signaler les ouvrages suivants (malheureusement ils sont tous publiés en allemand):

I) Antti Aarne, Estnische Märchen- und Sagenvarianten, I9I8 (= FF Communications 25), pp. II2-I39: légendes (IO7 types); pp. I39-I53: légendes étiologiques (8I types). Catalogue incomplet, mais très utile, contenant tous les types les plus importants.

2) Antti Aarne, Finnische Märchenvarianten, Ergänzungsheft I, I920 (= FFC 33), pp. 39-5I : légendes ; pp. 5I : légendes étiologiques. Sans grande importance ; contient quelques suppléments au système de FFC 25.

3) J. Qvigstad, Lappische Märchen- und Sagenvarianten, I925 (= FFC 60), pp. 3439: légendes étiologiques (63 types); pp. 39-62 : légendes (I68 types). Assez mauvais.

4) O. Loorits, Livische Märchen- und Sagenvarianten, I926 (= FFC 66), pp. 37-79: légendes (275 types); pp. 80-97: légendes étiologiques (I3 I types). Excellent.

5) J. R. W. Sinninghe, Katalog der niederlandischen Märchen-, Ursprungssagen-, Sagen- und Legendenvarianten, I943 (=FFC I32), pp. 47-52 : légendes étiologiques (48 types); pp. 52-I23 : légendes (plus de 500 types); pp. I24-I48 : légendes religieuses (plus de 200 types). Excellent; peut-être le modèle le plus recommandable pour vous.

Enfin un catalogue très spécial et très complet des légendes allemandes sur les géants.

6) V. Höttges, Typenverzeichnis der deutschen Reisen- und riesischen Teufelssagen, I937 (= FFC I22); 288 pages.

Il faut souligner qu'il n'y a pas de système international de numération des types de légendes (analogue à celui des contes populaires).

54. Serra i Boldú (1930-33). 
Encore une question (je n'ai pas de dictionnaire catalan sous les mains): qu'estce que veut dire le mot clatell (par exemple Rondallística p. 23I et 4I7)? peut-être «sommet de la tête » ou «fontanelle»?

En vous remerciant encore une fois, je reste votre très-dévoué. Walter Anderson.

[A-Am 19] 21.09.1957. Kiel, Forstweg 61. [lettre manuscrite]

Cher Collègue,

Hier j'ai reçu votre lettre du i8 septembre.

Quant aux trois ouvrages que vous désirez voir, je ne puis vous en envoyer mes propres exemplaires, car ceux-ci ont été anéanti (ainsi que tous mes livres et tous mes manuscrits) pendant le siège de Koenigsberg (I945); pourtant vous recevrez bientôt ces trois livres de la part du commissionnaire de l'Académie des Sciences de Finlande, et je vous prie de les accepter comme témoignage de ma reconnaissance.

Quant à l'ordre des légendes étiologiques, je vous conseille de suivre (en général) le système de Loorits (FFC 66) —d'ailleurs, ceux d'Aarne et de Sinninghe sont très semblables. Pour vous faciliter l'orientation, j'ai traduit les titres (non pas les résumés!) des 59 premiers types de Loorits, et j'ai donné le reste (type 60-I27) en abrégé.

Des légendes différentes concernant le même thème peuvent être distinguées par les lettres A, B, C, etc., par exemple: I. Création de la terre: A... B... C...

Votre très-dévoué. Walter Anderson.

$\underline{\text { Les légendes étiologiques }}^{55}$

(Système de Loorits, FFC 66)

\section{Légendes cosmogoniques}

I. La création de la terre; 2. L'origine des pierres; 3. Pourquoi les pierres sont devenues dures; 4 . Pourquoi les pierres ne croissent plus; 5 . Pourquoi l'eau de mer est salée; 6. L'origine du cours tortueux des rivières ; 7. Le goudronnement de la lune; 8-9. L'homme dans la lune; IO-II. L'origine de la Grande Ourse; I2. L'origine de la constellation Ceinture d'Orion ; I3. L'origine des vents et des tempêtes; I4. Le dialogue entre le froid et le chaud; I5. La guerre au ciel. [Anges et démons.]

\section{L'homme}

I6. La création de l'homme du reste de l'argile [par le diable]; I7. La création de la femme ; I8. L'origine du nœud de la gorge; I9. Pourquoi la forme de la nuque de l'homme est émoussée; 20 . L'origine des hommes chauves; 2I-22. Pourquoi le nombre des côtes est impair; 23-24. L'origine de la barbe et des cheveux ; 25. Pourquoi les cheveux de l'homme sont limités à quelques endroits définis; 26. L'origine de l'anus; 27. L'origine

55. Anderson inclut cette classification dans l'annexe à la lettre en forme de liste; mais dans cet article, la décision a été prise de présenter les éléments de classification dans une disposition horizontale. 
des seins de la femme; 28 . L'origine du scrotum; 29-30. L'origine des parties génitales; 3I. L'origine du coït; 32. L'origine des baisers; 33. Pourquoi le fiancé compte les doigts de la fiancée; 34 . Pourquoi Ève a la voix plus tendre qu'Adam.

35. La contagiosité du bâillement; 36. Pourquoi l'homme ne peut marcher aussitôt après sa naissance (comme les animaux); 37. Pourquoi l'homme ne sait plus le temps de sa mort; 38. L'origine du son nasal d'affirmation « mh »; 39. La généalogie des différents peuples; 40. La cuisson des langues. [C'est Dieu qui les cuit et les distribue aux différents peuples.]

\section{Légendes concernant la culture}

4I. L'origine des professions ; 42. Pourquoi les Juifs ne mangent pas de porc ; 43. Pourquoi Moïse avait la langue empesée ; 44. Origine du proverbe « Tout ce qui brille n'est pas de l'or »; 45. Origine des « enfants de Pharaon » [sirènes, phoques, etc.] ; 46. L'invention du piano ; 47. Pourquoi les hommes fument tant ; 48. Pourquoi les fumeurs crachent; 49. L'origine des bordels; 5O. L'origine de la levure; 5I. L'origine de l'ébuard; 52. L'origine de l'esprit de vin, de l'eau-de-vie, de la bière, du tabac, du jeu de cartes, des querelles et de l'égoïsme ; 53. L'origine de la bière ; 54. L'origine de l'eau-de-vie; 55. L'origine des querelles et des différends; 56. Pourquoi le diable est fâché contre les femmes; 57. Pourquoi le diable est fâché contre le forgeron ; 58. Pourquoi le diable ne fréquente pas l'étuve; 59. L'origine de l'Asa foetida; I30. L'origine de la Saint-Jean, de ses usages et du cri « ligo ».

Les mammifères

6o. Le chien; 6I. Le chat et le chien; 62. Le chat et la souris; 63-65. La vache; 66. La chèvre; 67. Le bélier; 68-70. Le cochon; 7I-72. Le lièvre; 7374. Le renard; 75 . Le cerf; 76 et I28. Le loup.

Les oiseaux

77. Le coucou; 78. Le rossignol et le coucou; 79. L'hirondelle; 8o. Le pic; 8I. La grive; 82. Le rouge-gorge; 83. La grive (le vanneau, le busard); 84. Le corbeau; 85-88. Comment les oiseaux ont appris leur chant; I29. La pipe.

Les poissons

89. L'anguille, la lamproie, le serpent; 90-94. La barbue; 95. La morue; 96. Le petit hareng de Suède; I3I. Les poissons en général.

Les insectes, les araignées, etc.

97. La fourmi et le fouille-merde; 98. La fourmi; 99. Le grillon; Ioo. L'araignée; IоI. La mouche; IO2. Le taon; IO3-IO4. La vermine; IO5. La puce; Io6. La puce, le pou, la lente

Les plantes

I07. Pourquoi les arbres ne parlent plus; Io8-IO9. Le tremble; IIO-III. Le bouleau; II2. Le bouleau et le pommier; II3-II4. Le sapin et le pin; II5. 
Le frêne; II6. L'aune; II7. La prunelle; II8. L'églantier ; II9. L'osier vert; I2O. Le houblon; I2I. La fève; I22. Le pois; I23. La pomme-de-terre; I24. L'airelle rouge; I25-I27. Le tabac.

(Comparez aussi les légendes $n^{\circ} 59$ et $\left.50 !\right)^{56}$

Addenda: no $128-$ I3I (voyez plus haut!) 57

[A-Am 20] 23.10.1957. Kiel. [lettre manuscrite]

Cher Collègue,

Ce soir j'ai reçu votre lettre du i9 octobre, et je me hâte d'y répondre.

I) Vraiment, c'est un scandale que Otto Harrassowitz à Wiesbade (le commissionnaire des FFC pour l'Allemagne) ne vous ait pas encore envoyé les numéros désirés. ${ }^{58}$ Je les avais commandés depuis plus d'un mois, et j'ai renouvelé mon ordre il y a huit jours, sans recevoir aucune réponse. Aujourd'hui je lui ai envoyé une troisième lettre, recommandée et très énergique, et j'espère d'obtenir enfin un résultat.

2) En parlant des animaux, des plantes, etc., vous devriez faire usage de la nomenclature française; ${ }^{59}$ s'il s'agit de termes rares et spéciaux qui manquent dans les dictionnaires, vous pouvez conserver le nom catalan, en y ajoutant entre deux parenthèses: (une espèce de...). Les noms latins scientifiques sont utiles, mais non pas indispensables.

3) Quant aux traditions sur des localités submergées ou englouties par la terre, il suffit de les registrer toutes ensemble, sans consacrer à chaque localité un numéro spécial.

4) Je n'ai pas l'intention de publier une édition revue de mon compte-rendu de votre Rondallística; ${ }^{60}$ j'en ferai la révision seulement pour compléter votre catalogue.

5) La préparation de la nouvelle édition d'Aarne-Thompson ${ }^{6 \mathrm{I}}$ exigera encore beaucoup de temps - plusieurs mois, au moins.

6) Je crois qu'il sera facile de trouver un éditeur pour votre catalogue tant désiré par les spécialistes. Moi, je ferai tout mon possible pour vous y aider.

7) Quant à vos Contes catalans, ${ }^{62}$ je regrette de ne pas les avoir encore reçu.

Votre très-dévoué. Walter Anderson.

56. Il semble qu'Anderson se réfère aux numéros 59 et 50 inclus dans la section «Légendes concernant la culture».

57. Anderson se réfère probablement au n ${ }^{\circ} 30$ inclus dans la liste de «Légendes concernant la culture».

58. Les Éditions Otto Harrassowitz établies à Wiesbaden n'avaient pas envoyé à Amades les livres de la FFC commandés par Anderson, et le folkloriste allemand se plaint du retard.

59. Ce détail prouve qu'Amades envisageait d'effectuer un catalogue en français.

60. Amades (I950).

6I. Aarne-Thompson (I96I).

62. Amades (1957a). 
Édition de la correspondance entre les folkloristes Walter Anderson et Joan Amades

[A-Am 21] 04-12-1957. Kiel. [carte postale manuscrite]

\section{Cher Collègue,}

J'ai reçu vos trois lettres et le précieux paquet de livres, dont je vous remercie de tout mon coeur.

Si je ne vous avais pas encore répondu, c'est que je voulais joindre a ma réponse au moins une partie des classifications que je vous ai promises. En peu de temps je vous renverrai vos fiches (je les ai déjà classifiées) en y ajoutant mes annotations aux contes de Serra i Boldú. Je regrette de ne pas avoir pu travailler si vite comme je l'aurais désiré.

Les numéros à l'astérisque se trouvent registrés dans le catalogue d'AarneThompson aux pages 2I4-252.

Les trois volumes des FFC sont un petit présent que je vous prie d'accepter de ma part.

Votre très-dévoué. Walter Anderson.

[A-Am 22] 16.12.1957. Kiel, Forstweg 61. [lettre manuscrite]

Cher Collègue,

Enfin je puis vous renvoyer vos I95 fiches (que j'ai numérotées au dos) avec mes classifications et remarques. ${ }^{63}$ J'y ajoute mes remarques sur les Rondalles populars et les Rondalles meravelloses de Serra i Boldú. ${ }^{64}$

Quelques jours avant de recevoir le volume des Contes catalans ${ }^{65}$ que vous avez eu la bonté de m'envoyer, j'en avais reçu un exemplaire de la part de l'éditeur. C'est ainsi que je possède maintenant deux exemplaires de votre précieux livre; si vous le permettez, j'en donnerai un au professeur Kurt Ranke (autrement je vous le renverrai).

Quant aux trois versions des « Trois oranges » publiées par Maspons i Labrós (I, p. 82-84; ${ }^{66} \mathrm{II}$, p. $52-56^{67}$ et III, p. I46-I50 $\left.{ }^{68}\right)$, j'en possède déjà des copies complètes.

Je ne sais pas selon quel modèle vous voulez arranger votre catalogue. Le système le plus utile serait celui que $\mathrm{M}$. Delarue a appliqué dans son grand catalogue en donnant des abrégés ou des analyses de toutes les versions de chaque type: mais ce système exigerait un immense travail et retarderait terriblement la publication du livre. C'est pourquoi je vous recommanderais de vous borner à de brefs résumés de chaque type, à la manière de ceux qui se trouvent dans le catalogue d'Aarne-Thompson (FF Communications 74), ${ }^{69}$ mais sans les analyses qui y occupent beaucoup de place (par exemple, p. 49, type 306 : I... II... III..., type 307 : I... II... III...).

63. Il s'agit d'un cahier de six pages intitulé « Remarques sur les contes qui manquent chez Aarne-Thompson », plus une page avec l'en-tête « (Amour et Psyché)».

64. Il s'agit de treize pages avec la classification des contes des dix-huit volumes des Rondalles populars (Serra i Boldú I930-33) et avec celle des contes contenus dans les Rondalles meravelloses (Serra i Boldú I924).

65. Amades (1957a).

66. « Les tres taronges de l'amor» (Maspons i871: 82-84).

67. « Les tres tarongetes» (Maspons I872: 52-56).

68. « La coloma blanca» (Maspons I875: I46-I50).

69. Aarne-Thompson (I928). 
Je vous avertis qu'un conte populaire dont une seule version est connue, est toujours suspect: c'est trop souvent une combinaison accidentelle de motifs traditionnels (ou non-traditionnels), une libre composition de quelque conteur (ou même de quelque collectionneur ou éditeur, c'est-à-dire une mystification). C'est pourquoi bien des types ajoutés par $\mathrm{M}$. Boggs ${ }^{70}$ au système d'Aarne-Thompson me paraissent assez douteux.

En vous remerciant encore une fois de tous vos beaux livres, je reste votre trèsdévoué. Walter Anderson.

[A-Am 23] 27.12.1957. Kiel. [lettre manuscrite]

\section{Cher Collègue,}

Aujourd'hui je puis enfin vous envoyer la classification du reste des recueils dont vous avez eu la bonté de me faire présent. ${ }^{7 \mathrm{I}}$

Est-ce que vous connaissez les recueils suivants (cités par Bolte et Polívka, vol. $\mathrm{V}$, p. 85), ${ }^{72}$ qui manquent dans la bibliographie de votre Rondallistica ${ }^{73}$

I) E. Contamine de Latour et R. Fouché-Delbosc: Contes espagnols (Paris, I889).

2) T. Baró: Cuentos del Ampurdán (I896).

3) F. Pelay y Briz: Cuentos populars catalans (date inconnue).

Et puis une prière nouvelle:

D’après votre résumé de la version inédite de « Maria Roseta » (celle de Josepa Moix) il me semble que cette version contient aussi (comme celle de Rondallística I73, mais non pas celle de l'Archiduc L. S., p. I8I-I85 ${ }^{74}$ les motifs de la transformation de la jeune fille en colombe et vice versa ; si c'est ainsi, je vous saurais gré d'une copie des passages de cette version inédite se rapportant à ces deux transformations.

Votre très-dévoué. Walter Anderson.

[A-Am 24] 30.01.1958. Kiel, Forstweg 61. [lettre manuscrite]

Cher Collègue,

Gran merci pour votre troisième paquet de livres et pour votre lettre du 6 janvier!

Aujourd'hui je vous renvoie vos fiches avec mes notes, de même que mes notes aux textes imprimés contenus dans votre troisième paquet. ${ }^{75}$ Je vous renvoie aussi

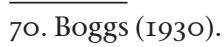

7I. Il s'agit de 6 pages contenant la classification des contes des collections et recueils suivants: «Cuentos del Rey Salomón» (Amades 1957c), «Rondalles de mai acabar» (Amades 1956), «El pecador regenerado y el anciano rejuvenecido (Amades I957b), Bibliothèque Ramon Llull, et les contes d'Amades «El manipig de pasta dolça » et «El viaje al infierno ».

72. Bolte-Polívka (I9I3-32).

73. Amades (I950).

74. « Maria Roseta (Amades I950: 4I6-420), « Es castell de ses roses » (Lluís Salvador I95I: I8I-I85).

75. Il s'agit de treize pages contenant les commentaires des contes manquant dans le catalogue Aarne-Thompson (I928). Les listes, qui correspondent aux fiches envoyées par Amades dans plusieurs paquets, sont identifiées respectivement par les lettres « $\mathrm{a} »$, « $\mathrm{b}$ » et « C » suivies du numéro d'ordre correspondant (a 7-a 37, b 2-b 98, et c I-c 80). 
le livret Els pèsols meravellosos ${ }^{76}$ dont vous m'avez régalé par méprise deux exemplaires.

Je ne vous conseillerais pas de registrer dans votre catalogue aussi les contes de Timoneda; pour ces contes, il y a déjà un livre spécial : J. WESLEY CHILDERS, Motif-Index of the Cuentos of Juan Timoneda, Bloomington, I948 (= Indiana University Publications, Folklore Series No 5 ). Il est vrai que ce livre est arrangé selon le système du Motif-Index de Thompson 77 (est non pas selon celui d'AarneThompson). ${ }^{78}$

Profitant de votre permission, j'ai cédé le second exemplaire de vos Contes Catalans $^{79}$ à M. le professeur Ranke, ${ }^{80}$ qui vous en remercie vivement.

Vous pouvez bien donner dans votre catalogue une place aux contes-légendes et même aux fables, si ces récits existent réellement dans la tradition orale catalane. Aarne et Thompson eux-mêmes ont admis dans leur registre beaucoup de fables ésopiques: AaTh 34, 4I, 5O, 5I, 57, 60, 6I, 62, 70, etc.

Les contes « apparentés avec... » doivent recevoir un numéro spécial (dans le voisinage des types nommés), si ce sont vraiment des types traditionnels, et non pas des récits estropiés casuellement.

Je regrette de ne pas avoir eu le temps de faire une révision générale de votre Rondallistica. ${ }^{8 \mathrm{I}} \mathrm{Je}$ la ferai certainement, et je vous en enverrai une copie.

Je n'ai rien envoyé à $M$. Thompson, ainsi que vous aurez à vous mettre en rapports avec lui personnellement.

Merci pour le résumé de la version inédite de « Maria Roseta »!

Votre très-dévoué. Walter Anderson.

P. S. J'ai beaucoup d'intérêt pour le troisième de vos contes de Reus (b 3) : L'Herba de quiquilimoqui. C'est ce type AaTh I360C, auquel j'ai consacré une monographie de 344 pages: Der Schwank vom alten Hildebrand (Dorpat, I93I). W. A. ${ }^{82}$

[A-Am 25] 11.04.1958. Kiel. [lettre manuscrite]

Cher Collègue,

Enfin je puis vous renvoyer (dans un paquet recommandé) vos dernières fiches accompagnées de mes annotations, ${ }^{83}$ de même que mes notes aux recueils d'Alcover (tome XVI), ${ }^{84}$ de Ferrer Ginard ${ }^{85}$ et de Maspons i Labrós (Tradicions del Vallès). ${ }^{86}$

76. Els pèsols meravellosos [1933].

77. Thompson (I932-36).

78. Aarne-Thompson (1928).

79. Amades (1957a).

8o. Kurt Ranke (Blankenburg am Harz, I4.04.I908 - Stadensen, O6.06.I985). Au sujet de la vie et de l'œuvre du folkloriste, consulter Nicolaisen (I989).

8I. Amades (I950).

82. Anderson (I93I).

83. Il s'agit des fiches f I-f 86 et k 5-k IO7 correspondant à « Récits qui manquent chez AaTh ».

84. Alcover (I936-72).

85. Ferrer Ginard [s.d.].

86. Maspons ([I876] I952). 
Je crois bien que vous êtes déjà très impatient à cause de mon long délai; mais j'ai dû achever un travail énorme en composant les notes comparatives pour le recueil des contes indiens de Tauscher (qui sera publié cette année), ${ }^{87}$ et j'ai dû écrire un article pour un livre qui se publiera en honneur de M. Archer Taylor. ${ }^{88}$ Je me sens bien coupable, mais vous aurez peut-être un peu de compassion pour moi en apprenant que depuis le mois de janvier je n'ai pas trouvé le temps pour continuer ma propre monographie sur les « Trois oranges ».

Je vous remercie de la copie du conte «L'herba de quiquilimoquis » qui est pour moi très intéressant.

Quant aux contes « apparentés ", « divergents », ou « comparables » à certains types d'Aarne-Thompson (il n'y a pas beaucoup de différence entre ces trois termes!), vous pouvez bien les registrer sous ces types (toujours avec une remarque spéciale!), ainsi comme je l'ai fait moi dans le compte-rendu de votre Rondallística ${ }^{89}$ (par exemple: 275. 277 (II 234), 306 ; vgl. [= comparez] 337 »). Pourtant, s'il s'agit d'un type bien distinct et constant (représenté par plusieurs textes), vous devrez lui donner un numéro à part ; par exemple, l'épisode du géant qui s'enlève ses propres intestins non pour pouvoir plus manger (comme dans AaTh Io88), mais pour mieux courir, pourrait recevoir le numéro * Io88B. Si vous y sentez quelques doutes, vous pouvez toujours recourir à l'arbitrage de M. Thompson.

Si un conte contient à côté d'épisodes mentionnés par AaTh quelques autres qui manquent dans leur catalogue, je registrerais ces derniers tout séparément: par exemple, je mentionnerais le conte Alcover XVI 5-46 non seulement sous les types 9IOA, IOOO, I048, I005, I085, I062, III5, I036, IOO2, IOO4, IO29, mais aussi sous les types nouveaux *IOO2B (semer sans ouvrir les sacs de semence), * IOO2C (manger et boire sans ouvrir les vases), * IO6OB (la pierre à chaux brisée), et * IO88B (s'enlever ses propres entrailles pour mieux courir).

Quant aux « Rondalles humanes » de la Rondallística ${ }^{\circ \circ}$ núm. 425-465 (et aussi à beaucoup d'autres contes qui les suivent dans votre recueil), il faudrait les enregistrer presque tous — comme vous le verrez dans la liste qui complétera mon compte-rendu; je vous ai promis cette liste et je vous l'enverrai plus tard, mais je ne puis le faire si tôt comme je l'aurais désiré.

On n'a pas le droit de laisser de côté des contes seulement à cause de leur brièveté; les contes les plus brefs sont parfois les plus intéressants et les plus précieux.

Toutes les légendes étiologiques, religieuses, de trésors, etc. doivent aussi être registrées, mais c'est une tâche spéciale et assez difficile. On peut bien publier un tel catalogue ensemble avec le catalogue des contes (comme vous pouvez le voir chez Loorits ${ }^{91}$ et chez Sinninghe ${ }^{92}$ ), mais on peut aussi en faire un livre à part.

Vous avez bien raison: il n'y a pas de limites bien distinctes entre les légendes et les contes proprement dits ; mais que faire ? Il faut toujours chercher la méthode la plus pratique pour celui qui fera usage de nos livres.

87. Il s'agit des notes sur le livre de Rudolf Tauscher (I959).

88. Anderson (1960 b).

89. Amades (I950).

90. Amades (I950).

9I. Loorits (1926).

92. Sinninghe (I943). 
C'est seulement aujourd'hui que j'ai reçu le troisième fascicule du premier volume de Fabula; et je ne sais pas, si beaucoup d'articles du second volume se trouvent déjà dans la typographie. Il me faudrait consulter le prof. Ranke que je vois assez rarement.

Mon compte-rendu de vos Contes catalans ${ }^{93}$ se trouve depuis longtemps entre les mains du rédacteur des Hessische Blätter für Volkskunde, mais il ne sera publié qu'à la fin de l'année courante (dans le nouveau volume de cette revue).

Avec les meilleurs souhaits votre très-dévoué. Walter Anderson.

[A-Am 26] 29.05.1958. Kiel, Forstweg 61. [lettre manuscrite]

\section{Cher Collègue,}

Je viens d'achever la révision de votre Rondallística; ${ }^{94}$ ainsi je puis enfin vous envoyer mes commentaires (45 pages). ${ }^{95}$

J'ai souligné en rouge les suppléments à mon compte-rendu et les émendations de ce dernier. ${ }^{96}$ Avant tout, je vous prie de corriger dans mon compte-rendu les lieux suivants:

page 59, ligne 6:[9B]: 260, 277 (II 234) ---- à biffer

page 59, ligne I3 d'en bas: $562: 220$---- à biffer

page 60 , ligne $5: 764$---- corriger en 765

page 60 , ligne 23 d'en bas:

$\underline{\mathrm{I} 653 \mathrm{~A}}$---- corriger en $\underline{\mathrm{I}} 653 \mathrm{~B}$

$\underline{\mathrm{I} 653^{*}}$---- corriger en $\underline{\mathrm{I} 654^{* *}}$

page 62 , ligne 16 , commencement $:\left(\mathrm{B}_{70} 8^{*} \underline{\mathrm{C}}\right)$---- corriger en $\left(\mathrm{B}_{70} 8^{*} \underline{\mathrm{A}}\right)$

page 62 , ligne 23, commencement: I424, I447, I45I ---- corriger en I424, I447, $\underline{\mathrm{I}} 452$

page 62, ligne 25, commencement: [II (II 2I6)] 60 [I63] ---- à biffer

page 62, ligne 12 d'en bas, fin: ZI5 ---- corriger en 7I6

page 62, ligne Io d'en bas: 276 [vgl. 604] ---- à biffer

A la fin du mois de juin j'espère de vous faire une visite à Barcelone. C'est que j'ai l'intention de me rendre à un congrès qui aura lieu à Porto du I8 au 23 juin (Colóquio de estudos etnográficos « Dr. José Leite de Vasconcelos »). J’y irai (si la situation politique le permettra) via Irún, mais en retournant je passerai par Barcelone, où j'ai l'intention de m'arrêter pour un ou deux jours. Je partirai de Porto pour l'Espagne le 24 juin; je serai accompagné par mon fils George, un lycéen de I8 ans que vous n'avez pas vu en I955 (il se trouvait alors dans un sanatorium).

Je vous prie de me faire savoir aussitôt que possible, si je pourrai vous voir à Barcelone vers le 24 ou 25 juin.

Avec les meilleurs souhaits votre très-dévoué. Walter Anderson.

93. Amades (1957a).

94. Amades (I950).

95. La lettre contient en annexe les 45 pages numérotées, avec l'indication dans cet ordre des informations suivantes: page de la Rondallística, numéro du conte, correspondance avec le volume et les pages de Les cent millors rondalles populars, numéro de catalogage AaTh et/ ou autres commentaires.

96. Anderson (I954b). 
[A-Am 27] 06.06.1958. Kiel. [carte postale manuscrite]

Cher Collègue,

Grand merci pour vos deux lettres du 30 mai et du I juin!

Votre article sera publié dans le 3 me fascicule de Fabula.${ }^{97}$ Vous en recevrez les épreuves probablement au mois d'août.

Je ne sais pas les noms des autres participants au congrès de Porto, $9^{98}$ mais je crois que Luís da Câmara Cascudo y viendra aussi, malgré la grande distance entre le Brésil et le Portugal.

Ainsi: au revoir à Porto!

Votre très-dévoué. Walter Anderson.

[A-Am 28] 22.07.1958. Kiel, Forstweg 61. [lettre manuscrite]

Cher Collègue,

Grand merci por votre lettre du i7 juillet et surtout pour la copie du conte de Milà i Fontanals!

J'espère que vous avez maintenant reçu aussi ma carte postale et le paquet de fiches que je vous ai envoyés le I6 juillet. ${ }^{99}$

Quant à ma révision de votre Rondallística, ${ }^{\text {Ioo }}$ je l'ai achevée dans les limites de ce qui a trouvé ou devra trouver son lieu dans le système d'Aarne-Thompson. Ce qui y manque sont:

I) d'innombrables anecdotes et petits contes plaisants sans grande diffusion, que seulement Loorits a tâché de registrer sans exception dans son catalogue des contes d'un peuple microscopique: les Livoniens (FF Communications 66); ${ }^{\text {IOI }}$

2) des légendes religieuses (en allemand Legenden), des légendes superstitieuses, locales, historiques, etc. (en allemand Sagen) et des légendes étiologiques (en allemand: Ursprungssagen), pour la numération desquelles il n'y a pas de systéme international. Si l'on veut les registrer (dans un catalogue spécial, publié ensemble avec le catalogue des contes proprement dits ou à part), on doit créer un système séparé pour chaque nation: c'est ce qu'ont fait Aarne pou les Estoniens (FFC 25: seulement un choix des légendes les plus répandues!), ${ }^{\text {IO2 }}$ Loorits pour les Livoniens (FF Communications 66) (103 $^{\mathrm{I}}$ et Sinninghe pour les Hollandais (FF Communications I32). ${ }^{104}$ C'est une tâche très intéressante et très utile, mais aussi extrêmement difficile et exigeant beaucoup de temps; je ne vous conseillerais pas de l'entreprendre à présent, ensemble avec le catalogue des contes proprement dits.

97. L'article d'Amades (I960) a été publié à titre posthume car le folkloriste est mort le I7 janvier 1959 .

98. Il s'agit du Colloque d'Études ethnographiques «Dr. José Leite de Vasconcelos » organisé à Porto (Portugal) du I8 au 23 juin I958 auquel se réfère Anderson dans sa lettre du 29 mai 1958.

99. Je n'ai trouvé dans la correspondance aucun document daté du I6 juillet I958 permettant d'identifier ces fiches mentionnées par Anderson.

IOO. Amades (1950).

IOI. Loorits (I926).

IO2. Aarne (I9I8).

IO3. Loorits (I926).

I04. Sinninghe (I943). 
Néanmoins, si vous désirez composer un tel catalogue des légendes catalanes, je vous conseillerais de créer un système plus ou moins analogue à celui de Sinninghe (dont le livre vous possédez). En tout cas, il est inutile d'envoyer les résumés de tous ces contes à M. Thompson.

Dans mon compte-rendu, les abbréviations «Aa $S$ » et «Aa US » sont expliquées à la page 6o, en bas:

«On sait que les légendes (agen) ne sont pas contenues dans le catalogue des types d'Aarne-Thompson, mais un registre des types de légendes les plus répandus se trouve dans FF Communications No 25 (Antti Aarne, Estnische Märchen- und Sagenvarianten, Hamina, I9I8). D'entre ceux, le recueil d'Amades contient les types suivants: Aa S Iq... (etc.).

«Ce qui est dit à l'égard des légendes (Sagen), est just aussi pour les contes étiologiques (Ursprungssagen): Aa US $3 . .$. (etc.) »

J'attends de votre part un nouveau paquet de fiches.

Je renouvelle la prière de m'envoyer le texte complet du conte: $\underline{\text { Sara Llorens de }}$ Serra, Rondallari de Pineda, № 54 « La Mora ». ${ }^{\text {I05 }}$

Je reste votre très-dévoué. Walter Anderson.

[A-Am 29] 30.10.1958. Kiel, Forstweg 61. [lettre manuscrite]

Cher collègue,

Je suis enchanté d'avoir reçu les neuf textes de contes que vous avez eu la bonté de m'envoyer. Je vous en remercie de tout mon cœur!

Il est vrai que les contes:

Els tres reis $(=120)$,

Les tres princeses tarongines $(=127)$, et

Els tres prínceps i les tres princeses germans

n'appartiennent pas au type AaTh 408: ils représentent un type tout spécial, dont l'existence m'avait été jusqu'à présent absolument inconnue. (Pourtant, il y a là une légère influence du type AaTh 408).

D'autre part, le conte 1 i 5 « Els set cavalls » contient toute la seconde partie du type « Les trois oranges »; je vous serais donc très obligé si vous pouviez m'envoyer aussi une copie de ce conte-là.

Je vous renvoie vos fiches 1 I-1 75 avec mes définitions et commentaires. ${ }^{\text {Io6 }}$

Les contes suivants sont sans aucun doute empruntés au recueil allemand des frères Grimm:

125 « la mà de l'albat »,

129 «El plato de madera »,

170 « La durada de la vida»,

172 «El sastre al cel»,

IO5. Il s'agit d'une version du conte «L'amor de les tres taronges » [ATU 408] incluse dans le Rondallari de Pineda, alors inédit et aujourd'hui publié par Josefina Roma (Llorens 2006).

Io6. Il s'agit des fiches correspondant à « Récits qui manquent chez AaTh». La lettre est accompagnée aussi de I3 pages contenant la classification des contes des recueils suivantes: « Rondalles » (Constans I952), "Rondalles gironines i valencianes » (Sales I95I), "Les rondalles populars catalanes » (Bertran I908-09), « Contes d'Eivissa » (Roure Torent I948), « Rondaies eivissenques » (Castelló Guasch I953); « Rondaies d'Eivissa » (Castelló Guasch I955), «Rondaies de Mallorca » (Lluís Salvador I95I). 
173 « Els quatre sabaters i la lluna »;

deux contes au recueil russe d'Afanasjev:

145 « El germà i la germana »,

174 «Els fills de la poma»,

et un conte à une chanson épique russe:

144 : «La sirena de la mar »

- Je vous remercie aussi du timbre-poste de 45 céntimos. ${ }^{107}$ Quant à l'émission courante (Franco en face), six timbres-poste manquant encore dans la collection de ma fille:

Io céntimos, I.4O pes., I.5O pes., I.80 pes., 2 pesetas (rouge, non pas lilas!), IO pesetas.

Je reste, avec les meilleurs souhaits, votre très-dévoué. Walter Anderson.

[A-Am 30] 04-11-1958. Kiel. [lettre manuscrite]

Cher collègue,

J'espère que vous avez reçu le paquet précedént (fiches 1 I-l 75) que je vous ai envoyé le 30 octobre.

Je vous renvoie les fiches $176-198$ qui sont arrivées ce matin. ${ }^{\text {108 }}$

Le conte 190 « La germana de l'isart » est une combinaison des types 450 ( «Le petit frère et la petite sœur ») et 408 ( «Les trois oranges »). Je serais donc très heureux si vous m'en pouviez envoyer une copie complète -de même qu'une copie du conte de Saturnino Calleja qui m'est absolument inconnu.

Je reste votre très-dévoué. Walter Anderson

[A-Am 31] 05.12.1958. Kiel, Forstweg 61. [lettre manuscrite]

Cher Collègue,

Merci pour votre brochure, pour les timbres-poste, pour les deux contes catalans, pour le conte galicien et pour le long conte « Los tres limones »!

Ce dernier conte n'est autre chose que la reproduction abrégée (et un peu modifiée) du conte français de Laboulaye « Les trois citrons ", ${ }^{\text {I09 }}$ qui de son côté est un remaniement du conte napolitain de Giambattista Basile « Le tre cetre ». ${ }^{\text {II }}$

C'est donc un conte littéraire ; mais j'ai beaucoup d'intérêt pour les versions littéraires des « Trois oranges »; c'est pourquoi je m'intéresse aussi pour le conte d'Octavio Muñoz « La portentosa historia de tres limones ». ${ }^{I I I}$ D'après ce que vous en écrivez, je suis prêt à croire que c'est une traduction complète du conte de Laboulaye: dans tous les points où le texte de Muñoz diffère du texte espagnol que

I07. Giambattista Basile (Naples, I575 - Giugliano/Campanie, 23.02.I632) a publié le conte «Le tre cetre » dans le livre Lo cunto de li cunti overo lo trattenemiento de peccerille [Il Pentamerone] (Basile I634-36). Au sujet de la vie et de l'œuvre de Basile, consulter Schenda (1977).

I08. Il s'agit des fiches correspondant à «Récits qui manquent chez AaTh ».

I09. Édouard Laboulaye (Paris, I8.0I.I8II - Paris, 25.05.I883) a publié le conte « Les trois citrons » dans le livre Contes bleus (Laboulaye I863).

IIo. Giambattista Basile (Naples, I575 - Giugliano/Campanie, 23.02.I632) a publié le conte «Le tre cetre » dans le livre Lo cunto de li cunti overo lo trattenemiento de peccerille [Il Pentamerone] (Basile I634-36). Au sujet de la vie et de l'œuvre de Basile, consulter Schenda (I977).

III. Muñoz [s.d.]. 
vous m'avez envoyé, il coïncide avec celui de cet écrivain français! —Il s’agit pour moi de trois questions:

I) Quand fut publié le conte de Muñoz?

2) Est-il vrai qu'il reproduit fidèlement le récit de Laboulaye?

3) Est-ce que le conte espagnol que vous m'avez envoyé a eu pour source le livret de Muñoz, ou bien directement le texte français de Laboulaye?

Pour résoudre les deux dernières questions, il me suffirait d'avoir sous les yeux une copie complète des deux premières pages du conte de Muñoz. J'ose donc encore une fois abuser de votre bonté et vous prier de m'envoyer cette copie.

M. Ranke a depuis longtemps reçu les épreuves que vous mentionnez.

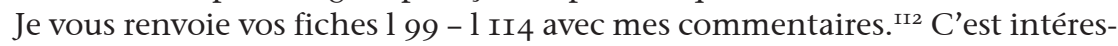
sant d'y trouver aussi le type AaTh I75, très rare en Europe (1 Io9 «En Mitja puça », la fin du récit).

Votre très-dévoué. Walter Anderson.

\subsection{Correspondance de Walter Anderson à Consol Mallofrè et Enrique- ta Mallofrè}

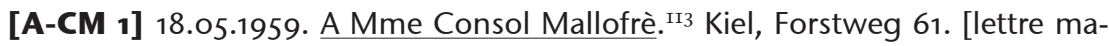
nuscrite]

Madame,

Je vous remercie vivement de votre aimable lettre, de la dernière lettre de feu votre beau-frère, de ses manuscrits, et surtout du grand dictionnaire catalan. C'est le meilleur présent que $\mathrm{M}$. Amades ait pu me faire : le seul dictionnaire catalan accessible à moi à Kiel, c'était un petit dictionnaire de poche qu'un collègue me prêtait de temps en temps.

Je comprends pleinement ce que vous et Mme Amades avez dû éprouver en recevant un tel coup du destin, et ce que vous en avez souffert et souffrez encore: même pour moi qui suis un étranger ce fut un coup de foudre. Il y a des hommes qui ne laissent dans la mémoire de ceux qui les ont connus que de beaux souvenirs: un tel homme, ce fut M. Amades.

Et c'est une grande perte non seulement pour vous, pour moi et pour tous ceux qui connaissaient votre beau-frère, mais aussi pour la science. ${ }^{\text {II } 4}$ Nous autres folkloristes, nous attendions avec curiosité et impatience le catalogue des contes catalans que M. Amades avait promis de composer. Je ne sais pas dans quel état ses préparatifs se trouvent; en tout cas il faut conserver avec le plus grand soin toutes les fiches contenant ses abrégés des contes - jusqu'au dernier feuillet. Dans la seconde moitié du mois d'août, nous aurons à Kiel le congrès international des investigateurs des contes populaires — ce congrès auquel (hélas!) M. Amades aurait

II2. Je n'ai pas trouvé dans la correspondance ces fiches mentionnées par Anderson.

II3. Anderson a adressé ses lettres à Enriqueta Mallofrè, veuve d'Amades, comme on peut le constater sur les enveloppes conservées dans la correspondance Anderson-Amades, même si les deux premières étaient adressées explicitement à Consol Mallofrè.

II4. Anderson fut très touché par la perte de son collègue et ami, et il en fait part dans l'article nécrologique qu'il écrivit pour le magazine Fabula (Anderson I96oa). 
dû jouer un grand rôle. Le prof. Stith Thompson y viendra aussi, et j'aurai l'occasion de lui parler du destin du catalogue catalan. ${ }^{\text {II5 }}$ Il faudra, peut-être, que vous nous envoyiez alors toutes les fiches de ce catalogue (et les autres papiers qui s'y rapportent); mais pour le présent je vous prie de ne pas le faire, pour ne pas exposer ces précieux manuscrits au risque d'être perdus. Quant au paquet de fiches que vous m'avez envoyé, je les garderai chez moi jusqu'au temps du congrès et je les montrerai au prof. Thompson. - La difficulté principale à rédiger ce catalogue, c'est l'impossibilité de trouver ailleurs qu'à Barcelone tous les livres et manuscrits cités par M. Amades (pour vérifier ses citations).

Quant au catalogue des légendes étiologiques, il y a moins de difficultés. Pourtant le manuscrit de M. Amades ne pourra pas être publié dans les FF Communications en catalan: c'est une série internationale où seulement trois langues sont admises —le français, l'anglais et l'allemand. Cette série est publiée en Finlande aux frais de l'Académie des Sciences Finnoise ; la Finlande a deux langues officielles - le finnois et le suédois; et cependant ni le finnois ni le suédois ne sont admis dans les FF Communications!

Si vous pouvez faire publier ce catalogue en catalan, ${ }^{116}$ tout va bien: mais alors il est indispensable de le publier ainsi comme feu votre beau-frère l'a rédigé —un bref abrégé de chaque conte et la liste complète de toutes les versions catalanes de ce conte.

A notre congrès, je parlerai à $\mathrm{M}$. Thompson aussi de ce catalogue des légendes étiologiques ; alors j'aurai besoin du manuscrit de M. Amades, mais pour le présent je vous prie de ne pas me l'envoyer.

L'écrit de M. Amades accepté par M. le prof. Ranke sera publié dans le premier fascicule de la revue Fabula de $1959,{ }^{117}$ et vous en recevrez les tirages à part.

J'ai l'honneur de vous saluer, et je vous prie de faire mes compliments respectueux à Mme Amades.

Je reste votre très-dévoué. Walter Anderson.

\section{[A-CM 2] 02.09.1959. A Mme Consol Mallofrè. Kiel. [lettre manuscrite]}

\section{Madame,}

Au Congrès international des investigateurs des contes populaires à Kiel et à Copenhague (I9.08.I959-29.08.I959) la question du catalogue des contes catalans, ainsi que celle des manuscrits et livres de M. Amades, fut discutée et, ensemble avec d'autres questions importantes, déléguée à un comité spécial. Préalablement, il fut constaté:

I) Pour rédiger le catalogue des contes catalans, il n'est pas assez d'avoir sous les yeux les fiches et les livres imprimés: c'est que sur les fiches les contes nonpubliés sont bien résumés (ainsi qu'on peut les reconnaître), mais sans indication des pages du manuscrit où l'on en peut trouver l'original.

2) Avant tout, il faudra donc ordonner les manuscrits originaux, les paginer et les conserver toujours dans le même ordre.

II5. Au sujet du projet de catalogage des contes catalans, interrompu avec la mort d'Amades, consulter Oriol (I999 et 200I).

II6.Je n'ai aucune preuve de la publication de ce catalogue.

II7. Malgré cette prévision, l'article d'Amades (I960) a été publié dans le deuxième fascicule du volume 3 du magazine Fabula. 
3) Puis il faut confronter toutes les fiches avec les originaux et marquer sur chaque fiche la page du manuscrit original.

4) Cette pagination et cette confrontation sont des tâches assez compliquées, et il faudra peut-être envoyer à Barcelone (pour quinze jours au moins) un spécialiste pour les exécuter.

5) On a pensé aussi à faire des microfilmes de tous les contes non-publiés de M. Amades, mais ce serait une entreprise assez coûteuse.

6) Quant aux recueils imprimés des contes populaires catalans qui se trouvent dans la bibliothèque de $\mathrm{M}$. Amades, ils devraient rester à Barcelone ainsi que les manuscrits originaux jusqu'au moment où le catalogue sera achevé. Si Mme votre sœur a l'intention de les vendre, je la prie de les vendre seulement à quelque institution barcelonaise accessible aux savants (par exemple à une bibliothèque publique), et aucunement à un libraire, afin que cette précieuse collection ne soit pas éparpillée.

7) Quant aux légendes étiologiques, il faut encore attendre si vous en trouverez un éditeur en Espagne.

8) A présent la tâche principale de vous et de Mme votre sœur sera celle de conserver tous les manuscrits et toutes les fiches de M. Amades, ainsi que tous les recueils de contes catalans qui se trouvent dans sa bibliothèque, sans que rien en soit perdu -202 fiches sont encore chez moi; je vous les renverrai plus tard.

Soyez persuadée que moi et les autres folkloristes, nous éprouvons trop d'intérêt pour cette affaire pour renoncer à sa réalisation.

Je reste votre très-dévoué. Walter Anderson.

\section{[A-EM 3] 21.12.1960. Kiel. [lettre manuscrite]}

Madame,

Grand merci pour votre lettre du I4 décembre et pour le recueil des contes galiciens que je viens de recevoir! M. Carré Alvarellos ${ }^{\mathrm{II} 8} \mathrm{~m}$ 'a déjà écrit une lettre, et deux de ses contes seront publiés dans l'annuaire de M. Hüllen. ${ }^{\text {II }}$

Les deux contes catalans que vous m'avez envoyés y seront publiés aussi —et (si vous y consentez) encore un troisième conte. ${ }^{\mathrm{I20}}$ C'est que M. Hüllen me dit il y a quelque temps, que vous ne lui aviez pas répondu; et je lui proposai alors de publier un bref conte extrêmement intéressant que feu votre mari avait eu la bonté de m'envoyer autrefois: L'herba de quiquilimoqui. Aujourd'hui j'en ai reçu la seconde épreuve.

J'ose donc m'adresser à vous et à Madame votre sœur avec les prières suivantes:

II8. Loís Carré Alvarellos est l'auteur d'un recueil de contes populaires galiciens publié dans plusieurs numéros de la Revista de Etnografia de Porto (Carré Alvarellos I963-67), avant d'être publié sous forme de livre (Carré Alvarellos I968).

II9. Georg Hüllen a été le secrétaire général d'une société dont l'objectif était la préservation des contes des peuples d'Europe, la Gesellschaft zur Pflege des Märchen-gutes der europäischen Völker.

I20. Anderson se réfère à l'œuvre de Kemminghausen-Hüllen (eds.) (I96I) au sujet de laquelle on peut voir le compte rendu fait par la folkloriste française Marie-Louise Tenèze (I962). 
I) de corriger un exemplaire de l'épreuve du texte catalan, si vous y trouvez des fautes d'impression (je vous prie d'en retenir l'autre exemplaire, de même que l'épreuve du texte allemand);

2) de traduire le texte de ma remarque française ${ }^{\mathrm{I2I}}$ en catalan et de m'envoyer cette traduction;

3) d'établir, si c'est possible, où et quand le conte «L'herba de quiquilimoqui » a été recueilli par M. Amades.

4) J'y ajoute une autre prière. Parmi les résumés de contes catalans que vous venez de m'envoyer il y en a un qui m'intéresse tout spécialement, puisque un de mes anciens disciples en a fait l'objet d'une monographie qu'il prépare : $\underline{\text { El }}$ príncep Picornell. Est-ce qu'il vous serait possible de trouver le texte complet de ce conte et de m'en envoyer une copie?

La nouvelle édition de l'index Aarne-Thompson n'a pas encore paru. Je tâcherai de régler l'affaire dont vous parlez directement avec $\mathrm{M}$. Thompson.

Je ferai savoir à M. Hüllen que vous avez trouvé le volume qui vous manquait.

Le livre suédois que vous mentionnez a pour sujet l'art populaire suédois. Le titre signifie : Peintures de la province de Dalarna et leurs modèles, par Svante Svärdström. Une étude sur la formation d'images populaires.

Avec les meilleurs souhaits pour vous et pour Madame votre sœur, votre trèsdévoué. Walter Anderson.

P.S. Merci beaucoup aussi pour les 49 timbres-poste!

\section{[Remarque]}

C'est une version catalane inédite de l'ancien conte Aarne-Thompson I360C, provenant de la collection du gran folkloriste catalan Joan Amades (I890-I959); le lieu et le temps de l'annotation son inconnus.

Ce conte fut composé (probablement au nord de la France) au plus tard au $\mathrm{I}^{\mathrm{e}}$ siècle, et se répandit presque dans toute l'Europe. Dans l'Espagne d'aujourd'hui il est extrêmement rare: je n'en connais que 2 versions espagnoles (d'Asturie), et en outre 3 portugaises (d'Alentejo et d'Algarve); en Amérique latine on en a recoueilli, à ce que je sais, une seule version, à savoir en Cuba: elle se trouve dans la collection inédite d'Herminio Portell Vilá, Cuentos populares cubanos, número 74 (je n'ai pas lu ce texte). D'autant plus intéressant est le fait que ce conte a été remanié déjà entre I7O2 et I742 par un écrivain espagnol depuis longtemps oublié, en forme d'intermède: Franciso de Castro, Entremés de los chirlos mirlos (imprimé, après la mort de l'auteur, à Madrid en I742). Comparez: Walter Anderson, Der Schwank vom alten Hildebrand, Dorpat, I93I (=Acta et Commentationes Universitatis Tartuensis [Dorpatensis] B 3I, I et B 33, I), pp. 46-47 et 98-IOI.

I2I. Le texte de la remarque auquel se réfère Anderson figure à la fin de la lettre. 
Édition de la correspondance entre les folkloristes Walter Anderson et Joan Amades

[A-EM 4] 14.02.1961. Kiel, Forstweg 61. [lettre manuscrite]

Madame,

En vous remerciant de votre aimable lettre du io janvier et de la correction des épreuves, je vous fais savoir que les trois contes catalans sont maintenant prêts pour l'impression. Le volume qui les contiendra doit paraître au mois de mai ou de juin. Il contiendra aussi deux contes galiciens de la collection de M. Carré Alvarellos que celui-ci a choisis lui-même; son recueil manuscrit que vous m'avez envoyé se trouve maintenant entre mes mains.

$\mathrm{Si}$, par hasard, vous trouverez le texte complet du conte «El Príncep Picornell », je serai très heureux; au cas contraire, le résumé détaillé que j'en possède me suffira.

Je suis toujours, Madame, votre très-dévoué. Walter Anderson.

\subsection{Correspondance d'Enriqueta Mallofrè et Consol Mallofrè à Helene} Anderson

[M-HA 1] 11.09.1962. Barcelona. [lettre tapée à la machine]

Mrs. Helen [sic.] Anderson. Kiel.

Dear Mrs. Anderson,

Your husband's death has been a terrible blow to us. We regret it as if it were one of our own family.

Science has had a great lost, since not every day can you find learned men as he was.

Fate is cruel and inexorable. We express you our deepest sorrow and sympathy on this moment of trial.

You can count as ever with the friendship of yours sincerely.

\section{Références bibliographiques}

AARne, Antti (I9I8) : Estnische Märchen- und Sagenvarianten. Folklore Fellows' Communications 25. Helsinki: Suomalainen Tiedeakatemia.

AARne, Antti; Stith ThOMPSON (I928) : Types of the folktale. Folklore Fellows' Communications 74. Helsinki: Suomalainen Tiedeakatemia.

- (I96I): The types of the folktale. Folklore Fellows' Communications I84. Helsinki: Suomalainen Tiedeakatemia.

Alcover, Antoni M. (I898): Aplech de rondaies mallorquines d'En Jordi des Recó, vol. 3. Ciutat de Mallorca: Tip. Católica de Sanjuan.

- (I936-72) : Aplec de rondaies mallorquines d'en Jordi des Recó. 24 vols. Ciutat de Mallorca: Moll.

— (I946): Les millors rondalles de Mallorca. Recueillis par En Jordi des Recó. Palma: Moll.

AmADES, Joan (I950) : Folklore de Catalunya: Rondallística (Rondalles-TradicionsLlegendes). Barcelona: Selecta. 
- (I948-49): Les cent millors rondalles populars. 2 vols. Barcelona: Selecta. [2ème éd. I953].

- (I956) : « Rondalles de mai acabar ». Revista de Ciencias Sociales del Instituto de Estudios Altoaragoneses no 25 (I956): 67-82.

- (I957a): Contes Catalans. Contes des cinq continents 3. París: Erasme.

- (I957b): «El pecador regenerado y el anciano rejuvenecido ». Revista del Centro de Lectura, 4ème époque, no 57 (I957): 26-30.

- (I957c) : «Cuentos del Rey Salomón. Contribución a los estudios universales del cuento ». Revista del Centro de Lectura, 4ème époque, no 59 (mai I957): 5255; nº 6I (juillet, I957): 77-80; nº 62 (août-septembre, I957): 88-90.

— (I960): «Les contes-devinettes de Catalogne ». Fabula 3 (2): I99-223.

ANDERSON, Walter (I93I) : Der Schwank vom alten Hildebrand. Eine vergleichende studie. Dorpat: K. Mattiesens buckdruckerei ant.-ges.

- (I95I): Ein Volkskundliches Experiment. Folklore Fellows' Communications I4I. Helsinki: Suomalainen Tiedeakatemia.

— (I953) : «Der türkische Marchenschatz ». Hessische Blätter für Volkskunde 44 (I953): III-I32.

- (I954a) : "Das sogenannte Märgen vom Eselmenschen ». Zeitschrift für Volkskunde 5I (I954): 2I5-236.

- (I954b) : «Eine katalanische Märchensammlung ». Schweizeriches Archiv für Volkskunde 50 (I954): 57-62.

- (I958): «Nochmals: Das sogenannte Märchen vom Eselmenschen ». Zeitschrift für Volkskunde 54 (I958): I2I-I25.

— (I960a): «Joan Amades ». Fabula 3 (I960): I86-I87.

- (I960b) : «Volkserzählungen in Tageszeitungen und Wochenblättern ». Dans Wayland D HAND; Gustave O. ARLT (éds.) : Humaniora. Essays in Literature, Folklore, Bibliography. Honoring Archer Taylor on His Seventieth Birthday. Locust Valley/New York: J.J. Augustin, p. 58-68.

[ATU] UTHER (2004).

BASILE, Giambattista (I634-36): Lo cunto de li cunti overo lo trattenemiento de peccerille [Pentamerone]. Naples: Ottavio Beltrano.

BERTRAN i Bros, Pau (I908-09): Les rondalles populars catalanes. Barcelona: Fidel Giró, 2 vols.

- (I909): El rondallari català. Barcelona: R. Miquel y Planas.

Boggs, Ralf R. (I930) : Index of Spanish folktales. Folklore Fellows' Communications 9o. Helsinki: Suomalainen Tiedeakatemia.

Bolte, Johannes; Georg PolívKA (I9I3-32) : Anmerkungen zu den Kinder- u. Hausmärchen der Brüder Grimm. Leipzig: Dieterich'sche Verlagsbuchhandlung, 5 vols.

CAlvo, Lluís (I990) : «Joan Amades i Gelats. Biografia ». Dans Antoni Anguela et alii El món de Joan Amades. Barcelona: Departament de Cultura de la Generalitat de Catalunya, p. III-I9I.

Carré Alvarellos, Loís (I963-67) : "Contos populares da Galiza ». Revista de Etnografia vols. I-IX. 
Édition de la correspondance entre les folkloristes Walter Anderson et Joan Amades

- (I968): Contos populares da Galiza. Porto: Junta Distrital.

CASTelló GuAsch, Joan (I953): Rondaies eivissenques. Palma: Impremta Successors d'en F. Ferrer.

- (I955): Rondaies d'Eivissa. Palma: Moll.

Chauvet, Horace (I947): Traditions populaires du Roussillon. Perpinyà: Imprimérie du Midi.

ConstAns, Lluís G. (I952): Rondalles. Banyoles: Impr. F. Granes.

ESPINOSA, Aurelio M. (I946-47): Cuentos populares españoles. 3 vols. Madrid: CSIC.

Els pèsols meravellosos [I933]: Biblioteca Ramon Llull. Barcelona: Impr. Elzeviriana i Llibr. Camí.

FERRER GINARD, Andreu [s.d.]: Rondaies populars de diferents autors i països., vol I. Artà: Tip. Cat. de A. Ferrer.

GUISCAFRÈ, Jaume (I999): «Una bibliografia de les edicions i les traduccions de les rondalles de mossèn Alcover ». Randa no 38 (I999): I5I-22I.

Honko, Lauri (I988): « Haavio, Martti ». Enzyklopädie des Märchens, vol. 6. Berlin: Walter de Gruyter, p. 348-350.

JÄRV, Risto (2008) : «Anderson, Walter (I885-I962) ». Dans Donald HAASE (éd.): The Greenwood Encyclopedia of Folktales \& Fairy Tales. Westport: Greenwood Press, p. 38-39.

LABoulaye, Édouard (I863): Contes bleus. Paris: G. Charpentier et cie.

Lluís SAlvador D'Àustria-Toscana (I95I): « Rondaies de Mallorca ». Dans Miquel Dolç (éd.): Obres completes. Barcelona: Selecta, p. I49-27I.

L'amor de les tres pomes. Rondalla [s.d.]. Biblioteca infantil. Barcelona: Estampa La Catalana.

Llorens, Sara (2006) : Rondallari de Pineda. Édité par Josefina Roma. Pineda de Mar: Ajuntament.

LOORITS, Oskar (I926) : Livische Märchen- und Sagenvarianten. Folklore Fellows' Communications 66. Helsinki: Suomalainen Tiedeakatemia.

Kemminghausen, Karl Schulte; Georg Hüllen (éds.) (I96I): Märchen der europäischen Völker. 2 vols. Münster: Aschendorff.

MuÑoz, Octavio [s.d.]: La portentosa historia de tres limones. Barcelona: Dalmau.

MAspons, Francesc de Sales (I880) : «Las bruixas ». Lo Gay Saber. Periódich literari quinzenal 3, 2ème époque, $\mathrm{n}^{\circ} 2$ I (I novembre, I880): 235-237; no 22 (I5 novembre, I880): 247-249; nº 23 (I décembre, I880): 259-26I; nº 24 (I2 décembre, I880): 27I-274.

— (I87I): Lo rondallayre, vol. I. Barcelona: Llibrería de Álvar Verdaguer.

— (I872): Lo rondallayre, vol. 2. Barcelona: Llibrería de Álvar Verdaguer.

— (I875): Lo rondallayre, vol. 3. Barcelona: Llibrería de Álvar Verdaguer.

— ([1876] I952): Tradicions del Vallès. Barcelona: Barcino.

Nicolaisen, W. F. H. (I989) : « Kurt Ranke and Einfache Formen ». Folklore Ioo, I (I989): II3-II9.

ORIOL, Carme (I998) : "Els rondallaris mallorquins, menorquins i eivissencs de l'Enzyklopädie des Märchens ». Estudis de llengua i literatura en honor de Joan Veny, vol. 2: 463-479. 
— (I999): «El projecte de catalogació de la rondallística catalana en els anys 50 ». Actes de l'Onzè Col-loqui Internacional de Llengua i Literatura Catalanes. Barcelona: Publicacions de l'Abadia de Montserrat, p. 365-386.

- (200I) : «Els primers treballs catalogràfics de les Rondaies Mallorquines d'en Jordi des Recó ». Estudis de llengua i literatura catalanes 43. Miscel-lània Giuseppe Tavani 2 : I85-20I.

— (2008): «Amades, Joan (I890-I959)». Dans Donald HAASE (éd.): The Greenwood Encyclopedia of Folktales \& Fairy Tales. Westport: Greenwood Press, p. 29-30.

OrIOL, Carme; Josep M. PUjOL (2003) : Índex tipològic de la rondalla catalana. Barcelona: Generalitat de Catalunya. Departament de Cultura.

- (2008): Index of Catalan Folktales. Folklore Fellows' Communications 294. Helsinki: Suomalainen Tiedeakatemia.

PENTIKÄINEN, Juha (I982) : «Folklore Fellows Communications ». Enzyklopädie des Märchens, vol. 4. Berlin: Walter de Gruyter, p. I4O3-I4O5.

RANKE, Kurt (I977): «Anderson, Walter ». Enzyklopädie des Märchens, vol. I. Berlin: Walter de Gruyter, p. 493-494.

RondCat: cercador de la rondalla catalana. Arxiu de Folklore. Departament de Filologia Catalana de la Universitat Rovira i Virgili <http://www.sre.urv.cat/ rondcat/> [Date de la requête: Mars 2OI4]

Roure Torent, Josep (I948): Contes d'Eivissa. Mèxic: Editorial Intercontinental.

SALES, Joan (I95I): Rondalles gironines i valencianes. Barcelona: Ariel.

SchendA, Rudolf (I977) : «Basile, Giambattista ». Enzyklopädie des Märchens, vol. I. Berlin: Walter de Gruyter, p. I296-I308.

SelJamaA, Elo-Hanna (2005) : «Walter Anderson: A Scientist beyond Historic and Geographic Borders ». Dans Kristin KuUtma; Tiiu JaAgo (éds.): Studies in Estonian Folkloristics and Ethnology: A Reader and Reflexive History. Tartu: Tartu University Press, p. I53-I68.

SERRA I BOLDÚ, Valeri (I924): Rondalles meravelloses. Barcelona: Editorial Catalana.

— (I930-33): Rondalles populars. I8 vols. Barcelona: Políglota.

SinNINGHE, Jacques R. W. (I943) : Katalog der niederländischen Märchen-, Ursprungssagen-, Sagen- und Legendenvarianten. Folklore Fellows' Communications I32. Helsinki: Suomalainen Tiedeakatemia.

TAUSCHER, Rudolf (I959) : Volksmärchen aus dem Jeyporeland. Berlin: Walter de Gruyter.

TenÈze, Marie-Louise (I957): «Paul Delarue (I889-I955) ». Fabula, I, I-2 : I62-I63.

— (I958) : «A la mémoire de Paul Delarue ». Arts et traditions populaires 6 (I958): 289-307.

— (I962): «Märchen der europäischen völker ». L'Homme 2 (I) (I962): I33-I35.

— (I98I): «Delarue, Paul-Alfred ». Enzyklopädie des Märchens, vol. 3, p. 385-387.

Thompson, Stith (I932-36): Motif-index of folk literature. 6 vols. Bloomington: Indiana University Press. [Deuxième édition corrigée et complétée I955-I958.]

UTHER, Hans-Jörg (2004) : The types of international folktales. Folklore Fellows' Communications 284-286. Helsinki: Suomalainen Tiedeakatemia. 